\title{
SPLIT-BY-NILPOTENT EXTENSIONS ALGEBRAS AND STRATIFYING SYSTEMS
}

\author{
M. LANZILOTTA, O. MENDOZA, C. SÁENZ.
}

\begin{abstract}
Let $\Gamma$ and $\Lambda$ be artin algebras such that $\Gamma$ is a split-bynilpotent extension of $\Lambda$ by a two sided ideal $I$ of $\Gamma$. Consider the socalled change of rings functors $G:={ }_{\Gamma} \Gamma_{\Lambda} \otimes_{\Lambda}-$ and $F:={ }_{\Lambda} \Lambda_{\Gamma} \otimes_{\Gamma}-$. In this paper, we find the necessary and sufficient conditions under which a stratifying system $(\Theta, \leq)$ in $\bmod \Lambda$ can be lifted to a stratifying system $(G \Theta, \leq)$ in $\bmod (\Gamma)$. Furthermore, by using the functors $F$ and $G$, we study the relationship between their filtered categories of modules; and some connections with their corresponding standardly stratified algebras are stated (see Theorem5.12 Theorem 5.15] and Theorem 5.18). Finally, a sufficient condition is given for stratifying systems in $\bmod (\Gamma)$ in such a way that they can be restricted, through the functor $F$, to stratifying systems in $\bmod (\Lambda)$.
\end{abstract}

\section{InTRODUCTION.}

Stratifying systems where introduced in [12, 20, 21, 27, 31] and developed in [16, 23, 24, 17, 26] with some applications, for example, in [10, 11, 13, 14, 15, 19, 22, 28,

Split-by-nilpotent extension algebras have been recently studied in various settings. For example, in almost split sequences [6], tilting modules [3] and quasi-tilted, laura, shod and weakly-shod algebras [5. We study these extension algebras from the point of view of the theory of stratifying systems.

The paper is organized as follows. After a brief section of preliminaries, we devote Section 3 to the study of the functors $F={ }_{\Lambda} \Lambda_{\Gamma} \otimes_{\Gamma}: \bmod (\Gamma) \rightarrow$ $\bmod (\Lambda)$ and $G={ }_{\Gamma} \Gamma_{\Lambda} \otimes_{\Lambda}-: \bmod (\Lambda) \rightarrow \bmod (\Gamma)$, where $\Gamma$ is a split-bynilpotent extension of $\Lambda$ by a two sided ideal $I$ of $\Gamma$.

In Section 4 , we show that, for any $M \in \bmod (\Lambda)$, the algebra $\operatorname{End}_{\Gamma}(G M)$ is always an split-by-nilpotent extension of $\operatorname{End}_{\Lambda}(M)$ by $\operatorname{Hom}_{\Lambda}(M, I \otimes M)$ (see Theorem 4.3).

The section 5 is the main section in the paper. We give necessary and sufficient conditions such that the image under $G$, of a stratifying system in $\bmod (\Lambda)$, is a stratifying system in $\bmod (\Gamma)$. Here, the main results are 5.12, 5.15, 5.18

2010 Mathematics Subject Classification. Primary 16G10. Secondary 18G99. The authors thank the financial support received from Project PAPIIT-UNAM IN100810. 
and 5.19, Finally, in Section 6, we give a sufficient condition (see 6.4) for stratifying systems in $\bmod (\Gamma)$ in such a way that they can be restricted, through the functor $F$, to stratifying systems in $\bmod (\Lambda)$.

\section{Preliminaries.}

Throughout this paper, the term algebra means artin algebra over a commutative artin ring $R$. For an algebra $\Lambda$, the category of finitely generated left $\Lambda$-modules is denoted by $\bmod (\Lambda)$. We denote by $\operatorname{proj}(\Lambda)$ the full subcategory of $\bmod (\Lambda)$ whose objects are the projective $\Lambda$-modules. Unless otherwise specified, all the modules are finitely generated. Furthermore, for any positive integer $t$, we set $[1, t]:=\{1,2, \cdots, t\}$.

Definition 2.1. 12, 20, Let $\Lambda$ be an algebra. A stratifying system $(\Theta, \leq)$, of size $t$ in $\bmod (\Lambda)$, consists of a family of indecomposable $\Lambda$-modules $\Theta=$ $\{\Theta(i)\}_{i=1}^{t}$ and a linear order $\leq$ on the set $[1, t]$, satisfying the following two conditions.

(a) $\operatorname{Hom}_{\Lambda}(\Theta(i), \Theta(j))=0$ if $i>j$.

(b) $\operatorname{Ext}_{\Lambda}^{1}(\Theta(i), \Theta(j))=0$ if $i \geq j$.

For a set $\Theta$ of $\Lambda$-modules, let $\mathfrak{F}(\Theta)$ be the subcategory of $\bmod (\Lambda)$ consisting of the $\Lambda$-modules $M$ having a $\Theta$-filtration, that is, a sequence of submodules $0=M_{0} \subseteq M_{1} \subseteq \cdots \subseteq M_{s}=M$ such that each factor $M_{i+1} / M_{i}$ is isomorphic to a module in $\Theta$ for all $i$.

Definition 2.2. 21] Let $\Lambda$ be an algebra. An Ext-projective stratifying system $(\Theta, Q, \leq)$, of size $t$ in $\bmod (\Lambda)$, consists of two families of non-zero $\Lambda$-modules $\Theta=\{\Theta(i)\}_{i=1}^{t}$ and $\underline{Q}=\{Q(i)\}_{i=1}^{t}$, with $Q(i)$ indecomposable for all $i$, and $a$ linear order $\leq$ on the set $[1, t]$, satisfying the following three conditions.

(a) $\operatorname{Hom}_{\Lambda}(\Theta(i), \Theta(j))=0$ if $i>j$.

(b) For each $i \in[1, t]$, there is an exact sequence

$$
\varepsilon_{i}: 0 \longrightarrow K(i) \longrightarrow Q(i) \stackrel{\beta_{i}}{\longrightarrow} \Theta(i) \longrightarrow 0,
$$

with $K(i) \in \mathfrak{F}(\{\Theta(j): j>i\})$.

(c) $Q$ is $\Theta$-projective. That is $\operatorname{Ext}_{\Lambda}^{1}(Q, \boldsymbol{\Theta})=0$, where $Q:=\oplus_{i=1}^{t} Q(i)$ and $\Theta:=\oplus_{i=1}^{t} \Theta(i)$.

Recall that (see [21, Corollary 2.13]) an Ext-projective stratifying system $(\Theta, \underline{Q}, \leq)$ gives the stratifying system $(\Theta, \leq)$. Furthermore, for a given a stratifying system $(\Theta, \leq)$, we know by [21, Corollary 2.15] that there is a unique, up to isomorphism, Ext-projective stratifying system $(\Theta, \underline{Q}, \leq)$. So, it is said that $(\Theta, \underline{Q}, \leq)$ is the Ext-projective stratifying system associated to the stratifying system $(\Theta, \leq)$. We also have the dual notion of the Ext-injective stratifying system $(\Theta, \underline{Y}, \leq)$ associated to the stratifying system $(\Theta, \leq)$ (see [12, 20, 21]). 
The stratifying systems are related with the so-called standardly stratified algebras and so we introduce this notion. Let $\Lambda$ be an algebra. For $M, N \in$ $\bmod (\Lambda)$, the trace $\operatorname{Tr}_{M}(N)$ of $M$ in $N$, is the $\Lambda$-submodule of $N$ generated by the images of all morphisms from $M$ to $N$.

We next recall the definition (see [29, 9, 2, 7]) of the class of standard $\Lambda$-modules. Let $n$ be the rank of the Grothendieck group $K_{0}(\Lambda)$. We fix a linear order $\leq$ on the set $[1, n]$ and a representative set ${ }_{\Lambda} P=\left\{{ }_{\Lambda} P(i): i \in\right.$ $[1, n]\}$, containing one module of each iso-class of indecomposable projective $\Lambda$-modules. Observe, that the set ${ }_{\Lambda} P$ determines the representative set ${ }_{\Lambda} S=$ $\left\{{ }_{\Lambda} S(i)\right\}_{i=1}^{n}$ of simple $\Lambda$-modules, where ${ }_{\Lambda} S(i):={ }_{\Lambda} P(i) / \operatorname{rad}\left({ }_{\Lambda} P(i)\right)$ for each $i$.

The set of standard $\Lambda$-modules is ${ }_{\Lambda} \Delta=\left\{{ }_{\Lambda} \Delta(i): i \in[1, n]\right\}$, where ${ }_{\Lambda} \Delta(i)={ }_{\Lambda} P(i) / \operatorname{Tr}_{\oplus_{j}>i \Lambda} P(j)\left({ }_{\Lambda} P(i)\right)$. Then, ${ }_{\Lambda} \Delta(i)$ is the largest factor module of ${ }_{\Lambda} P(i)$ with composition factors only amongst ${ }_{\Lambda} S(j)$ for $j \leq i$. The algebra $\Lambda$ is said to be a standardly stratified algebra, with respect to the linear order $\leq$ on the set $[1, n]$, if $\operatorname{proj}(\Lambda) \subseteq \mathfrak{F}\left({ }_{\Lambda} \Delta\right)$ (see [2, 7, 8]). In this case, it is also said that the pair $(\Lambda, \leq)$ is a standardly stratified algebra (or an ss-algebra for short).

Let $\Lambda$ be an algebra and $\leq$ be a linear order on $[1, n]$, where $n=r k K_{0}(\Lambda)$. By [9], it follows that the pair $(\Lambda \Delta, \leq)$ is always a stratifying system (it is known as the canonical stratifying system). Furthermore, if $(\Lambda, \leq)$ is an ss-algebra, the representative set of the indecomposable projective $\Lambda$-modules ${ }_{\Lambda} P=\left\{{ }_{\Lambda} P(i)\right\}_{i=1}^{n}$ satisfies that the triple $\left({ }_{\Lambda} \Delta,{ }_{\Lambda} P, \leq\right)$ is the Ext-projective stratifying system associated to $(\Lambda \Delta, \leq)$.

The main connection between Ext-projective stratifying systems and the class of ss-algebras is given by the following result.

Theorem 2.3. 21] Let $(\Theta, Q, \leq)$ be an Ext-projective stratifying system of size $t$ in $\bmod (\Lambda), \Gamma=\operatorname{End}_{\Lambda}(Q)^{o p}, H=\operatorname{Hom}_{\Lambda}(Q,-): \bmod (\Lambda) \rightarrow \bmod (\Gamma)$ and $L=Q \otimes_{\Gamma}-: \bmod (\Gamma) \rightarrow \bmod (\Lambda)$. Then, the following statements hold true.

(a) The family ${ }_{\Gamma} P=\{H(Q(i)): i \in[1, t]\}$ is a representative set of the indecomposable projective $\Gamma$-modules. In particular, $\Gamma$ is a basic algebra and $r k K_{0}(\Gamma)=t$.

(b) $(\Gamma, \leq)$ is an ss-algebra, that is, $\operatorname{proj}(\Gamma) \subseteq \mathfrak{F}(\Gamma \Delta)$.

(c) The restriction $\left.H\right|_{\mathfrak{F}(\Theta)}: \mathfrak{F}(\Theta) \rightarrow \mathfrak{F}\left({ }_{\Gamma} \Delta\right)$ is an exact equivalence of categories and $\left.L\right|_{\mathfrak{F}\left({ }_{\Gamma} \Delta\right)}: \mathfrak{F}\left({ }_{\Gamma} \Delta\right) \rightarrow \mathfrak{F}(\Theta)$ is a quasi-inverse of $\left.H\right|_{\mathfrak{F}(\Theta)}$.

(d) $H(\Theta(i)) \simeq{ }_{\Gamma} \Delta(i)$, for all $i \in[1, t]$.

(e) add $(Q)=\mathfrak{F}(\Theta) \cap^{\perp} \mathfrak{F}(\Theta)$, where $M \in^{\perp} \mathfrak{F}(\Theta)$ if and only if the restriction functor $\left.\operatorname{Ext}_{\Lambda}^{1}(M,-)\right|_{\mathfrak{F}(\Theta)}=0$. 
Another nice feature, for a stratifying system $(\Theta, \leq)$ of size $t$, is that an analogous of the Jordan-Holder's Theorem holds for the set of "relative simples" $\Theta$ in $\mathfrak{F}(\Theta)$. That is, for any $M \in \mathfrak{F}(\Theta)$ and all $i \in[1, t]$, the filtration multiplicity $[M: \Theta(i)]$ is well defined (see [21, Lemma $2.6(\mathrm{c})]$ ). Therefore, we have the so-called $\Theta$-length $\ell_{\Theta}(M):=\sum_{i=1}^{t}[M: \Theta(i)]$ of $M$.

In what follows, we introduce some features about split-by-nilpotent extensions.

Definition 2.4. [3, 28] Let $\Gamma$ and $\Lambda$ be algebras, and let $I$ be a two-sided ideal of $\Gamma$. It is said that $\Gamma$ is a split-by-nilpotent extension of $\Lambda$ by $I$, if $I \subseteq \operatorname{rad}(\Gamma)$ and there is an exact sequence of abelian groups

$$
0 \longrightarrow I \longrightarrow \Gamma \stackrel{\pi}{\longrightarrow} \Lambda \longrightarrow 0
$$

such that $\pi$ is an epimorphism of algebras and there is a morphism of algebras $\sigma: \Lambda \rightarrow \Gamma$ with $\pi \sigma=1_{\Lambda}$.

In all that follows, we fix the two algebras $\Lambda$ and $\Gamma$, and the two-sided ideal $I \unlhd \Gamma$, such that $\Gamma$ is a split-by-nilpotent extension of $\Lambda$ by $I$. Observe that the morphisms of algebras $\sigma: \Lambda \rightarrow \Gamma$ and $\pi: \Gamma \rightarrow \Lambda$, induce in a natural way (change of rings), a bimodule structure on $I, \Gamma$ and $\Lambda$. Furthermore, $\Gamma=\Lambda \oplus I$ as abelian groups, and the multiplicative structure of $\Gamma$ can be seen as $\gamma_{1} \gamma_{2}=\left(\lambda_{1}, i_{1}\right)\left(\lambda_{2}, i_{2}\right)=\left(\lambda_{1} \lambda_{2}, i_{1} \lambda_{2}+\lambda_{1} i_{2}+i_{1} i_{2}\right)$; in this case $\pi(\lambda, i)=\lambda$ and $\sigma(\lambda)=(\lambda, 0)$.

We also remark that in [1], the authors consider a quotient path algebra $\Gamma$ and give sufficient conditions on a set of arrows $\mathcal{A}$ of the ordinary quiver of $\Gamma$, so that $\Gamma$ is a split-by-nilpotent extension of $\Lambda:=\Gamma / I$ by $I$, where $I$ is the ideal of $\Gamma$ generated by the set $\mathcal{A}$.

Remark 2.5. The morphisms of algebras $\sigma: \Lambda \rightarrow \Gamma$ and $\pi: \Gamma \rightarrow \Lambda$ have the following properties.

(a) $\pi$ is a morphism of $\Gamma-\Gamma$ bimodules. In particular, we have the exact sequence of $\Gamma-\Gamma$ bimodules

$$
0 \longrightarrow{ }_{\Gamma} I_{\Gamma} \longrightarrow{ }_{\Gamma} \Gamma_{\Gamma} \stackrel{\pi}{\longrightarrow}{ }_{\Gamma} \Lambda_{\Gamma} \longrightarrow 0
$$

(b) $\pi$ and $\sigma$ are morphisms of $\Lambda-\Lambda$ bimodules. In particular

$$
{ }_{\Lambda} \Gamma_{\Lambda}={ }_{\Lambda} \Lambda_{\Lambda} \bigoplus{ }_{\Lambda} I_{\Lambda}
$$

as $\Lambda-\Lambda$ bimodules.

(c) ${ }_{\Lambda} \Gamma_{\Gamma} \otimes_{\Gamma \Gamma} \Lambda_{\Lambda} \simeq{ }_{\Lambda} \Lambda_{\Lambda} \simeq{ }_{\Lambda} \Lambda_{\Gamma} \otimes_{\Gamma \Gamma} \Gamma_{\Lambda}$ as $\Lambda-\Lambda$ bimodules.

Lemma 2.6. Let $M \in \bmod (\Gamma)$ and consider ${ }_{\Lambda} M$ as $\Lambda$-module given by the change of rings $\sigma: \Lambda \rightarrow \Gamma$. Then, there exist natural isomorphisms

$$
\operatorname{Hom}_{\Gamma}\left({ }_{\Gamma} \Gamma_{\Lambda}, \Gamma_{\Gamma} M\right) \simeq{ }_{\Lambda} M \simeq \operatorname{Hom}_{\Gamma}\left({ }_{\Gamma} \Lambda_{\Lambda},{ }_{\Gamma} M\right)
$$


Proof. It is straightforward to see that the natural morphisms

$$
\varphi_{M}: \operatorname{Hom}_{\Gamma}\left({ }_{\Gamma} \Gamma_{\Lambda}, \Gamma_{\Gamma} M\right) \rightarrow{ }_{\Lambda} M \text { and } \psi_{M}:{ }_{\Lambda} M \rightarrow \operatorname{Hom}_{\Gamma}\left({ }_{\Gamma} \Lambda_{\Lambda},{ }_{\Gamma} M\right),
$$

given by $\varphi_{M}(f):=f(1)$ y $\psi_{M}(m)(\lambda)=\sigma(\lambda) m$, are isomorphisms of $\Lambda$ modules such that $\psi_{M}^{-1}(f)=f(1)$ and $\varphi_{M}^{-1}(m)(\gamma)=\gamma m$.

Lemma 2.7. $\operatorname{Hom}_{\Lambda}\left({ }_{\Lambda} \Lambda_{\Gamma},{ }_{\Lambda} \Lambda_{\Gamma}\right) \simeq{ }_{\Gamma} \Lambda_{\Gamma}$ as $\Gamma-\Gamma$ bimodules.

Proof. The morphism $\varphi: \operatorname{Hom}_{\Lambda}\left({ }_{\Lambda} \Lambda_{\Gamma},{ }_{\Lambda} \Lambda_{\Gamma}\right) \rightarrow_{\Gamma} \Lambda_{\Gamma}$, given by $\varphi(f):=f(1)$ is an isomorphism of $\Gamma-\Gamma$ bimodules with inverse $\varphi^{-1}(\lambda)(x)=x \lambda$.

\section{The usual Change of Rings Functors}

Let $\Gamma$ be a split-by-nilpotent extension of $\Lambda$ by $I \unlhd \Gamma$. We have the functors

$$
\bmod (\Gamma) \stackrel{F}{\longrightarrow} \bmod (\Lambda) \stackrel{G}{\longrightarrow} \bmod (\Gamma),
$$

where $F:={ }_{\Lambda} \Lambda_{\Gamma} \otimes_{\Gamma}-$ and $G:={ }_{\Gamma} \Gamma_{\Lambda} \otimes_{\Lambda}-$. These functors are known as change of rings functors.

We also recall, that a functor $H: \mathcal{A} \rightarrow \mathcal{B}$, between additive categories, reflects zero objects if $H(A)=0$ implies that $A=0$ for any $A \in \mathcal{A}$. Furthermore, for a given class $\mathcal{X}$ of objects in $\mathcal{A}$, the essential image of the functor $\left.H\right|_{\mathcal{X}}: \mathcal{X} \rightarrow \mathcal{B}$, which is denoted by $\operatorname{Im}\left(\left.H\right|_{\mathcal{X}}\right)$ or $H(\mathcal{X})$, is the full subcategory of $\mathcal{B}$ whose objects are all the objects $Z \in \mathcal{B}$ for which there is an object $X \in \mathcal{X}$ such that $Z \simeq H(X)$.

In the following lemma, we write down some well-known basic properties (see [3, 5, 25]), and for the convenience of the reader, it is included a proof.

Lemma 3.1. For the change of rings functors $F$ and $G$, the following statements hold true.

(a) $F G \simeq 1_{\bmod (\Lambda)}$.

(b) The functors $F$ and $G$ are faithful. In particular, they reflect zero objects.

(c) For any $M \in \bmod (\Lambda)$, the $\Gamma$-module $G(M)$ is indecomposable if and only if $M$ is indecomposable.

(d) For any $N \in \bmod (\Gamma)$, if the $\Lambda$-module $F(N)$ is indecomposable then $N$ is indecomposable.

Proof. (a) It follows from 2.5 (c).

(b) The fact that $G$ is faithful follows from (a). Let us prove that $F$ is also faithful. Indeed, for any $M \in \bmod (\Gamma)$, it can be seen easily that $\varphi_{M}:{ }_{\Lambda} \Lambda_{\Gamma} \otimes_{\Gamma} M \rightarrow{ }_{\Lambda} M$, given by $\varphi_{M}(\lambda \otimes m):=\sigma(\lambda) m$, is an isomorphism of $\Lambda$-modules, where ${ }_{\Lambda} M$ has the structure of $\Lambda$-module given by the change of rings $\sigma: \Lambda \rightarrow \Gamma$. Furthermore, for any $f: M \rightarrow N$ in $\bmod (\Gamma)$, we have the 
following commutative diagram

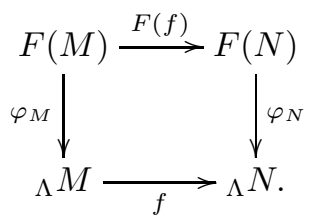

Thus, if $F(f)=0$ then $f=0$; proving that $F$ is faithful.

(c) Let $M \in \bmod (\Lambda)$ be such that $G(M)$ is indecomposable. In particular, $M \neq 0$. If $M=M_{1} \oplus M_{2}$ then $G(M)=G\left(M_{1}\right) \oplus G\left(M_{2}\right)$; and since $G(M)$ is indecomposable, we have that $G\left(M_{1}\right)=0$ or $G\left(M_{2}\right)=0$. Thus, by (b), it follows that $M_{1}=0$ or $M_{2}=0$; proving that $M$ is indecomposable.

Let $M \in \bmod (\Lambda)$ be an indecomposable $\Lambda$-module. So $M \neq 0$ and by (b) $G(M) \neq 0$. If $G(M)=N_{1} \oplus N_{2}$ then by (a) $M=F\left(N_{1}\right) \oplus F\left(N_{2}\right)$. Therefore, using that $M$ is indecomposable, it follows that $F\left(N_{1}\right)=0$ or $F\left(N_{2}\right)=0$. Thus, by (b), we have that $N_{1}=0$ or $N_{2}=0$; and so $G(M)$ is indecomposable.

(d) As in (c), the item (d) follows from the fact that $F$ reflects zero objects.

Lemma 3.2. Let $I_{\Lambda}$ be a projective $\Lambda$-module. Then, the following statements hold true.

(a) $\Gamma_{\Lambda}$ is a projective $\Lambda$-module and $\left.\operatorname{Tor}_{1}^{\Gamma}\left(\Lambda_{\Gamma},-\right)\right|_{\operatorname{Im}(G)}=0$.

(b) For all $n \geq 0$ and any $X, Y \in \bmod (\Lambda)$, we have that

$$
\operatorname{Ext}_{\Gamma}^{n}(G(X), G(Y)) \simeq \operatorname{Ext}_{\Lambda}^{n}(X, Y) \oplus \operatorname{Ext}_{\Lambda}^{n}\left(X, I \otimes_{\Lambda} Y\right) .
$$

Proof. (a) Since $\Gamma_{\Lambda}=\Lambda_{\Lambda} \oplus I_{\Lambda}$ (see2.5(b)), it follows that $\Gamma_{\Lambda}$ is a projective $\Lambda$-module, and hence $\operatorname{Tor}_{1}^{\Lambda}\left(\Gamma_{\Lambda},-\right)=0$. In particular, we get that $G$ is an exact functor.

Let $X \in \operatorname{Im}(G)$. Then there is an isomorphism $f: G(M) \rightarrow X$ for some $M \in \bmod (\Lambda)$. Consider an exact sequence ending at $M$, that is, $\eta: 0 \rightarrow$ $K \rightarrow P \stackrel{h}{\rightarrow} M \rightarrow 0$. Since $G$ is an exact functor, we get the exact sequence $\eta^{\prime}: 0 \rightarrow G(K) \rightarrow G(P) \stackrel{f G(h)}{\rightarrow} X \rightarrow 0$. Thus, by applying the functor $F$ to $\eta^{\prime}$ and using 3.1 (a), we get an exact and commutative diagram

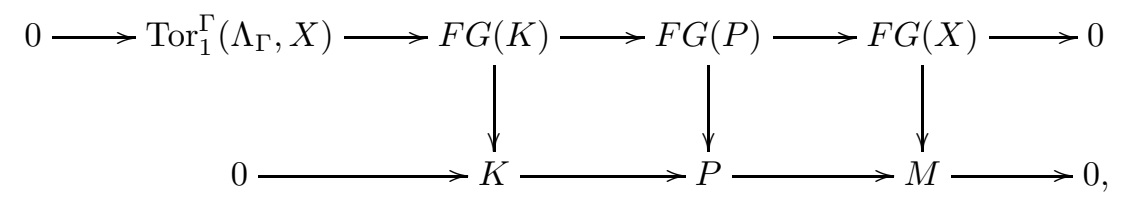

where the vertical arrows are isomorphisms; proving that $\operatorname{Tor}_{1}^{\Gamma}\left(\Lambda_{\Gamma}, X\right)=0$. 
(b) Since $\Gamma_{\Lambda}$ is projective, by [30, Exercise 9.21], we get that

$$
\operatorname{Ext}_{\Gamma}^{n}(G(X), G(Y)) \simeq \operatorname{Ext}_{\Lambda}^{n}\left(X, \operatorname{Hom}_{\Gamma}\left({ }_{\Gamma} \Gamma_{\Lambda}, G(Y)\right)\right) .
$$

Thus, the result follows from 2.5 (b) and 2.6

Theorem 3.3. For the change of rings functors $F$ and $G$, the following statements hold true.

(a) The restriction functor $\left.F\right|_{\operatorname{Im}(G)}: \operatorname{Im}(G) \rightarrow \bmod (\Lambda)$ is an equivalence and $G: \bmod (\Lambda) \rightarrow \operatorname{Im}(G)$ is a quasi-inverse. Moreover, if $I_{\Lambda}$ is projective, then $\left.F\right|_{\operatorname{Im}(G)}$ and $G$ are exact functors.

(b) add $(G M) \subseteq \operatorname{Im}(G)$ for any $M \in \bmod (\Lambda)$. Thus, the restriction functor $\left.F\right|_{\operatorname{add}(G M)}: \operatorname{add}(G M) \rightarrow \operatorname{add}(M)$ is an equivalence and $\left.G\right|_{\operatorname{add}(M)}: \operatorname{add}(M) \rightarrow \operatorname{add}(G M)$ is a quasi-inverse.

Proof. (a) Let $\varepsilon: F G \rightarrow 1_{\bmod (\Lambda)}$ be the isomorphism of functors given in 3.1 (a). Firstly, we assert that $\left.F\right|_{\operatorname{Im}(G)}$ is full. Indeed, let $X, Y \in \operatorname{Im}(G)$. Then there are isomorphisms $\alpha_{1}: X \rightarrow G(M)$ and $\alpha_{2}: Y \rightarrow G(N)$ for some $M, N \in \bmod (\Lambda)$. We need to show that $F: \operatorname{Hom}_{\Gamma}(X, Y) \rightarrow \operatorname{Hom}_{\Lambda}(F X, F Y)$ is surjective. For any $f \in \operatorname{Hom}_{\Lambda}(F X, F Y)$, set $\bar{f}:=F\left(\alpha_{2}\right) f F\left(\alpha_{1}^{-1}\right), f^{\prime}:=$ $\varepsilon_{N} \bar{f} \varepsilon_{M}^{-1}$ and $h:=\alpha_{2}^{-1} G\left(f^{\prime}\right) \alpha_{1}$. So an straightforward calculation gives us that $F(h)=f$, proving that $\left.F\right|_{\operatorname{Im}(G)}$ is full. Observe, that $\left.F\right|_{\operatorname{Im}(G)}$ is dense since $F G \simeq 1_{\bmod (\Lambda)}$. Moreover, by 3.1 (b) it follows that $\left.F\right|_{\operatorname{Im}(G)}$ is an equivalence. Furthermore, since $F G \simeq 1_{\bmod (\Lambda)}$, we conclude that $G: \bmod (\Lambda) \rightarrow \operatorname{Im}(G)$ is a quasi-inverse of $\left.F\right|_{\operatorname{Im}(G)}$.

Finally, assume that $I_{\Lambda}$ is projective. Then, by 3.2 (a), we get that $\left.F\right|_{\operatorname{Im}(G)}$ and $G$ are exact functors.

(b) Let $M \in \bmod (\Lambda)$ and let $X \in \operatorname{add}(G M)$. Then, there exists $Z \in$ $\bmod (\Gamma)$ such that $X \oplus Z=G M^{m}$, for some $m$. Since $G$ is an additive functor, we may assume that $X$ is indecomposable. Thus, from the Krull-RemakSchmidt Theorem and 3.1 (c), we get that $X \simeq G M^{\prime}$ for some indecomposable direct summand $M^{\prime}$ of $M$ and thus $X \in \operatorname{Im}(G)$.

Corollary 3.4. The restriction functor $\left.F\right|_{\operatorname{proj}(\Gamma)}: \operatorname{proj}(\Gamma) \rightarrow \operatorname{proj}(\Lambda)$ is an equivalence and $\left.G\right|_{\operatorname{proj}(\Lambda)}: \operatorname{proj}(\Lambda) \rightarrow \operatorname{proj}(\Gamma)$ is a quasi-inverse.

Proof. It follows from 3.3 (b) since $G\left({ }_{\Lambda} \Lambda\right) \simeq{ }_{\Gamma} \Gamma$ and $\operatorname{add}\left({ }_{\Gamma} \Gamma\right)=\operatorname{proj}(\Gamma)$.

\section{The FunCtor $G$ And SPlit-BY-NILPOTENT EXtensions}

We recall that the term algebra means artin $R$-algebra over a commutative artinian ring $R$ and $\Gamma$ is a split-by-nilpotent extension of $\Lambda$ by $I$. Consider the change of rings functor $G:={ }_{\Gamma} \Gamma_{\Lambda} \otimes_{\Lambda}-: \bmod (\Lambda) \rightarrow \bmod (\Gamma)$. Recall that ${ }_{\Lambda} \Gamma_{\Lambda}={ }_{\Lambda} \Lambda_{\Lambda} \bigoplus_{\Lambda} I_{\Lambda}$ as bimodules. Hence $\Gamma \otimes_{\Lambda} N=\left(\Lambda \otimes_{\Lambda} N\right) \oplus\left(I \otimes_{\Lambda} N\right)$ as $\Lambda$-modules. For the sake of simplicity, we some times write $(M, N)$ instead of $\operatorname{Hom}_{\Lambda}(M, N)$. 
For each pair $M, N \in \bmod (\Lambda)$, we consider the isomorphism $\delta=\delta_{M, N}$ of $R$-modules

$$
\operatorname{Hom}_{\Lambda}(M, N) \oplus \operatorname{Hom}_{\Lambda}(M, I \otimes N) \stackrel{\delta}{\longrightarrow} \operatorname{Hom}_{\Gamma}(G M, G N),
$$

which is obtained as the composition $\delta:=\tau \circ \operatorname{Hom}_{\Lambda}\left(M, \varphi^{-1}\right) \circ \nu$ of the following isomorphism of $R$-modules:

(a) $\nu:(M, N) \oplus(M, I \otimes N) \rightarrow(M, \Lambda \otimes N) \oplus(M, I \otimes N)=(M, \Gamma \otimes N)$, where $\nu(f, g):=(\bar{f}, g)$ and $\bar{f}(m):=1 \otimes f(m)$ for all $m \in M$.

(b) $\operatorname{Hom}_{\Lambda}\left(M, \varphi^{-1}\right):(M, \Gamma \otimes N) \rightarrow\left(M, \operatorname{Hom}_{\Gamma}(\Gamma, G N)\right)$, where

$$
\varphi^{-1}\left(\gamma_{1} \otimes n\right)\left(\gamma_{2}\right)=\gamma_{2}\left(\gamma_{1} \otimes n\right)=\gamma_{2} \gamma_{1} \otimes n
$$

as can be seen from the proof of 2.6

(c) $\tau:\left(M, \operatorname{Hom}_{\Gamma}(\Gamma, G N)\right) \rightarrow \operatorname{Hom}_{\Gamma}(\Gamma \otimes M, G N)=\operatorname{Hom}_{\Gamma}(G M, G N)$, where $\tau(\alpha)(\gamma \otimes m)=\alpha(m)(\gamma)$.

Proposition 4.1. $\delta=(G, L):(M, N) \oplus(M, I \otimes N) \rightarrow \operatorname{Hom}_{\Gamma}(G M, G N)$ as a matrix, where $L(g)(\gamma \otimes m)=\gamma g(m)$. Furthermore $L: \operatorname{Hom}_{\Lambda}(M, I \otimes N) \rightarrow$ $\operatorname{Hom}_{\Gamma}(G M, G N)$ is a monomorphism.

Proof. Consider the natural inclusion $i_{1}:(M, N) \rightarrow(M, N) \oplus(M, I \otimes N)$. We assert that $\delta i_{1}=G$. Indeed, let $f \in(M, N), \gamma \in \Gamma$ and $m \in M$. So we have $\delta i_{1}(f)(\gamma \otimes m)=\tau\left(\varphi^{-1} \bar{f}\right)(\gamma \otimes m)=\left(\varphi^{-1} \bar{f}(m)\right)(\gamma)=\gamma(1 \otimes f(m))=$ $\gamma \otimes f(m)=G(f)(\gamma \otimes m)$.

Let $i_{2}:(M, I \otimes N) \rightarrow(M, N) \oplus(M, I \otimes N)$ be the natural inclusion. We check that $\delta i_{2}=L$. Indeed, let $g \in(M, I \otimes N), \gamma \in \Gamma$ and $m \in M$. So we have $\delta i_{2}(g)(\gamma \otimes m)=\left(\tau \varphi^{-1}(0, g)\right)(\gamma \otimes m)=\varphi^{-1}(0, g)(m)(\gamma)=\gamma g(m)$.

Finally, let $y \in \operatorname{Hom}_{\Lambda}(M, I \otimes N)$ be such that $L(y)=0$. Then $\delta\left(\begin{array}{l}0 \\ y\end{array}\right)=$ $G(0)+L(y)=0$ and since $\delta$ is an isomorphism, it follows that $y=0$; proving that $L$ is a monomorphism.

Corollary 4.2. Let $M, N \in \bmod (\Lambda)$. Then, the following conditions are equivalent.

(a) $G: \operatorname{Hom}_{\Lambda}(M, N) \rightarrow \operatorname{Hom}_{\Gamma}(G M, G N)$ is an isomorphism.

(b) $\operatorname{Hom}_{\Lambda}(M, I \otimes N)=0$.

Proof. Consider the natural inclusion $i_{1}:(M, N) \rightarrow(M, N) \oplus(M, I \otimes N)$. Then, by 4.1, we know that $\delta i_{1}=G$ and thus the result follows since $\delta$ is an isomorphism.

Theorem 4.3. For any $M \in \bmod (\Lambda)$, the algebra $\operatorname{End}_{\Gamma}(G M)$ is an split-bynilpotent extension of $\operatorname{End}_{\Lambda}(M)$ by $\operatorname{Hom}_{\Lambda}(M, I \otimes M)$.

Proof. Let $M \in \bmod (\Lambda)$. By 4.1, we have the exact sequence of $R$-modules

$$
0 \longrightarrow \operatorname{Hom}_{\Lambda}(M, I \otimes M) \stackrel{L}{\longrightarrow} \operatorname{End}_{\Gamma}(G M) \stackrel{\vartheta}{\longrightarrow} \operatorname{End}_{\Lambda}(M) \longrightarrow 0,
$$


where $\vartheta:=\pi_{1} \delta^{-1}$ and $\pi_{1}: \operatorname{End}_{\Lambda}(M) \oplus \operatorname{Hom}_{\Lambda}(M, I \otimes M) \rightarrow \operatorname{End}_{\Lambda}(M)$ is the canonical projection. Observe that $G: \operatorname{End}_{\Lambda}(M) \rightarrow \operatorname{End}_{\Gamma}(G M)$ is a ring morphism and $\vartheta G$ is the identity map.

We assert that $\vartheta: \operatorname{End}_{\Gamma}(G M) \rightarrow \operatorname{End}_{\Lambda}(M)$ is a ring morphism. Indeed, we firstly transfer, by using the isomorphism $\delta$, the multiplicative structure of the ring $\operatorname{End}_{\Gamma}(G M)$ to the $R$-module $\operatorname{End}_{\Lambda}(M) \oplus \operatorname{Hom}_{\Lambda}(M, I \otimes M)$. That is, for any $\alpha=\left(f_{\alpha}, g_{\alpha}\right)$ and $\beta=\left(f_{\beta}, g_{\beta}\right)$ in $\operatorname{End}_{\Lambda}(M) \oplus \operatorname{Hom}_{\Lambda}(M, I \otimes M)$, we set $\alpha \beta:=\delta^{-1}(\delta(\alpha) \delta(\beta))$. In what follows, we shall compute the above product and show that

$$
(*) \quad \delta(\alpha) \delta(\beta)=G\left(f_{\alpha} f_{\beta}\right)+L\left(g_{\alpha} f_{\beta}+\varepsilon\right) .
$$

Indeed $\delta(\alpha) \delta(\beta)=G\left(f_{\alpha}\right) G\left(f_{\beta}\right)+L\left(g_{\alpha}\right) G\left(f_{\beta}\right)+L\left(g_{\alpha}\right) L\left(g_{\beta}\right)+G\left(f_{\alpha}\right) L\left(g_{\beta}\right)$. But $L\left(g_{\alpha}\right) G\left(f_{\beta}\right)=L\left(g_{\alpha} f_{\beta}\right)$ since $L\left(g_{\alpha}\right) G\left(f_{\beta}\right)(\gamma \otimes m)=L\left(g_{\alpha}\right)\left(\gamma \otimes f_{\beta}(m)\right)=$ $\gamma g_{\alpha}\left(f_{\beta}(m)\right)=L\left(g_{\alpha} f_{\beta}\right)(\gamma \otimes m)$. To compute $\mu:=L\left(g_{\alpha}\right) L\left(g_{\beta}\right)+G\left(f_{\alpha}\right) L\left(g_{\beta}\right)$, we proceed as follows. Observe that $\mu=\left(G\left(f_{\alpha}\right)+L\left(g_{\alpha}\right)\right) L\left(g_{\beta}\right)=\delta(\alpha) L\left(g_{\beta}\right)$. Consider the morphism $\left(0, g_{\beta}\right): M \rightarrow G M$. Using the fact that $I \unlhd \Gamma$, it can be seen that $\operatorname{Im}\left(\delta(\alpha)\left(0, g_{\beta}\right)\right) \subseteq I \otimes M$. Thus, the morphism $\varepsilon$, which is the composition of $M \rightarrow \operatorname{Im}\left(\delta(\alpha)\left(0, g_{\beta}\right)\right) \subseteq I \otimes M$, satisfies that $L(\varepsilon)=$ $\mu$. Indeed, $L(\varepsilon)(\gamma \otimes m)=\gamma \varepsilon(m)=\gamma \delta(\alpha)\left(0, g_{\beta}\right)(m)=\gamma \delta(\alpha)\left(g_{\beta}(m)\right)=$ $\delta(\alpha)\left(\gamma g_{\beta}(m)\right)=\delta(\alpha)\left(L\left(g_{\beta}\right)(\gamma \otimes m)\right)=\delta(\alpha) L\left(g_{\beta}\right)(\gamma \otimes m)$; proving $(*)$. Now, we are ready to prove that $\vartheta: \operatorname{End}_{\Gamma}(G M) \rightarrow \operatorname{End}_{\Lambda}(M)$ is a ring homomorphism. That is, by $(*)$, we have that $\vartheta(\delta(\alpha) \delta(\beta))=\pi_{1} \delta^{-1}(\delta(\alpha) \delta(\beta))=$ $f_{\alpha} f_{\beta}=\pi_{1} \delta^{-1} \delta(\alpha) \pi_{1} \delta^{-1} \delta(\beta)=\vartheta(\delta(\alpha)) \vartheta(\delta(\beta))$.

Finally, we prove that $\operatorname{Im}(L) \subseteq \operatorname{rad}\left(\operatorname{End}_{\Gamma}(G M)\right)$. To see that, it is enough to check that the ideal $\operatorname{Im}(L)$ is nilpotent. Let $g_{1}, g_{2}, \cdots, g_{n} \in \operatorname{Hom}_{\Lambda}(M, I \otimes$ $M), \gamma \in \Gamma$ and $m \in M$. Since $L\left(g_{1}\right) L\left(g_{2}\right) \cdots L\left(g_{n}\right)(\gamma \otimes m) \in I^{n} \otimes M$; and using the fact that $I$ is nilpotent, it follows that $\operatorname{Im}(L)$ is also nilpotent.

\section{EXTENDing STRATIFying Systems With the FUnCtor $G$}

In this section, we consider a split-by-nilpotent extension $\Gamma$ of $\Lambda$ by $I$. As we have seen before, there is the change of rings functor $G:={ }_{\Gamma} \Gamma_{\Lambda} \otimes_{\Lambda}-$ : $\bmod (\Lambda) \rightarrow \bmod (\Gamma)$. We give conditions for the image under $G$, of a stratifying system in $\bmod (\Lambda)$, to be a stratifying system in $\bmod (\Gamma)$.

Definition 5.1. A stratifying system $(\Theta, \leq)$, of size $t$ in $\bmod (\Lambda)$, is compatible with the ideal $I \unlhd \Gamma$ if the following conditions hold.

(C1) $\operatorname{Hom}_{\Lambda}\left(\Theta(j), I \otimes_{\Lambda} \Theta(i)\right)=0$ for $j>i$.

(C2) $\operatorname{Ext}_{\Lambda}^{1}\left(\Theta(j), I \otimes_{\Lambda} \Theta(i)\right)=0$ for $j \geq i$.

Proposition 5.2. Let $\Theta=\{\Theta(i)\}_{i=1}^{t}$ be objects in $\bmod (\Lambda)$ and $\leq$ be a linear order on $[1, t]$. If $I_{\Lambda}$ is projective, then the following conditions are equivalent.

(a) $(G(\Theta), \leq)$ is a stratifying system in $\bmod (\Gamma)$.

(b) $(\Theta, \leq)$ is a stratifying system in $\bmod (\Lambda)$, which is compatible with the ideal I. 
Proof. By 3.1, we know that the functor $G: \bmod (\Lambda) \rightarrow \bmod (\Gamma)$ reflects and preserves indecomposables. On the other hand, since $I_{\Lambda}$ is projective, we have by 3.2 that

$$
\operatorname{Ext}_{\Gamma}^{i}(G(X), G(Y)) \simeq \operatorname{Ext}_{\Lambda}^{i}(X, Y) \oplus \operatorname{Ext}_{\Lambda}^{i}\left(X, I \otimes_{\Lambda} Y\right),
$$

for all $X, Y \in \bmod (\Lambda)$ and any $i$. Thus, the equivalence between (a) and (b) follows.

Corollary 5.3. Let $\Theta=\{\Theta(i)\}_{i=1}^{t}$ be objects in $\bmod (\Lambda)$, and let $\leq$ be a linear order on $[1, t]$. If ${ }_{\Lambda} I_{\Lambda} \in \operatorname{add}\left({ }_{\Lambda} \Lambda_{\Lambda}\right)$ then the following conditions are equivalent.

(a) $(G(\Theta), \leq)$ is a stratifying system in $\bmod (\Gamma)$.

(b) $(\Theta, \leq)$ is a stratifying system in $\bmod (\Lambda)$.

Proof. Let ${ }_{\Lambda} I_{\Lambda} \in \operatorname{add}\left({ }_{\Lambda} \Lambda_{\Lambda}\right)$. Then $I \otimes_{\Lambda} X \in \operatorname{add}(X)$ for any $X \in \bmod (\Lambda)$. Therefore, any stratifying system in $\bmod (\Lambda)$ is compatible with the ideal $I$. So, the result follows from 5.2 .

Let us consider the following examples.

Example 5.4. Consider the trivial extension $\Gamma:=\Lambda \ltimes I$ of an algebra $\Lambda$ by its minimal injective cogenerator $I:=D(\Lambda)$. If $\Lambda$ is a symmetric algebra, it is well known that ${ }_{\Lambda} I_{\Lambda} \simeq{ }_{\Lambda} \Lambda_{\Lambda}$ as bimodules. Therefore, in this case, the needed hypothesis in 5.3 holds. We recall that in [12] stratifying systems for symmetric special biserial algebras are constructed.

Example 5.5. Let $Q$ be the quiver $\bullet^{3} \stackrel{\beta}{\rightarrow} \bullet^{1} \stackrel{\alpha}{\rightarrow} \bullet^{2}$. Consider the quotient path $k$-algebra $\Gamma:=k Q /\langle\alpha \beta\rangle$ with the ideal $I:=\langle\bar{\beta}\rangle \unlhd \Gamma$. Then $\Gamma$ is a split-bynilpotent extension of $\Lambda:=\Gamma / I$ by $I$. Moreover, the ordinary quiver $Q_{\Lambda}$ of $\Lambda$ is $\bullet^{3} \bullet \bullet^{1} \stackrel{\alpha}{\rightarrow} \bullet^{2}$. We consider the natural order $1 \leq 2 \leq 3$. Then, we have the canonical stratifying system $\left({ }_{\Lambda} \Delta, \leq\right)$ in $\bmod (\Lambda)$ where ${ }_{\Lambda} \Delta(i)={ }_{\Lambda} S(i)$ is the simple $\Lambda$-module associated to the vertex $i \in Q_{\Lambda}$. It can be seen that $I_{\Lambda} \simeq e_{3} \Lambda$ (where $e_{3}$ is the primitive idempotent associated with the vertex 3 ), $I \otimes_{\Lambda} \Delta(1)=0=I \otimes_{\Lambda} \Delta(2)$ and $I \otimes_{\Lambda} \Delta(3) \simeq{ }_{\Lambda} \Delta(1)$. Thus, the stratifying system $\left({ }_{\Lambda} \Delta, \leq\right)$ is compatible with the ideal $I$; and so by 5.2 it follows that $\left(G\left({ }_{\Lambda} \Delta\right), \leq\right)$ is a stratifying system in $\bmod (\Gamma)$.

As we have seen in 5.2 the notion of stratifying system compatible with the ideal $I$ plays an important role. In the following proposition, we give conditions for the canonical stratifying system to be compatible with the ideal $I$. For doing so, let $\left.{ }_{\Lambda} P={ }_{\Lambda} P(i)\right\}_{i=1}^{n}$ be a representative set of the indecomposable projective $\Lambda$-modules, where $n:=r k K_{0}(\Lambda)$, and let $\leq$ be a linear order on the set $[1, n]:=\{1,2, \cdots, n\}$. Let us consider the set of standard $\Lambda$-modules ${ }_{\Lambda} \Delta$, computed by using the pair $\left({ }_{\Lambda} P, \leq\right)$, and also the representative set ${ }_{\Lambda} S=\left\{{ }_{\Lambda} S(i)\right\}_{i=1}^{n}$ of simple $\Lambda$-modules, where ${ }_{\Lambda} S(i):=$ ${ }_{\Lambda} P(i) / \operatorname{rad}\left({ }_{\Lambda} P(i)\right)$ for each $i$. Recall that each ${ }_{\Lambda} \Delta(i)$ has composition factors 
only amongst ${ }_{\Lambda} S(j)$ with $j \leq i$. That is, the multiplicity $\left[{ }_{\Lambda} \Delta(i):{ }_{\Lambda} S(j)\right]$ of the simple ${ }_{\Lambda} S(j)$ in ${ }_{\Lambda} \Delta(i)$ is equal to zero for $j>i$. So, we start with the following definition.

Definition 5.6. The pair $\left({ }_{\Lambda} S, \leq\right)$ is admissible with the ideal $I \unlhd \Gamma$ if

$$
\left[I \otimes{ }_{\Lambda} S(i):{ }_{\Lambda} S(j)\right]=0 \quad \text { for } \quad j>i .
$$

Lemma 5.7. Let $I_{\Lambda}$ be a projective $\Lambda$-module. Then, the following statements are equivalent.

(a) The pair $\left({ }_{\Lambda} S, \leq\right)$ is admissible with the ideal $I$.

(b) $\left[I \otimes{ }_{\Lambda} \Delta(i):{ }_{\Lambda} S(j)\right]=0$ for $j>i$.

Proof. (a) $\Rightarrow$ (b) It follows from the fact that $I \otimes_{\Lambda}-: \bmod (\Lambda) \rightarrow \bmod (\Lambda)$ is an exact functor and ${ }_{\Lambda} \Delta(i) \in \mathfrak{F}\left(\left\{{ }_{\Lambda} S(t): t \leq i\right\}\right)$ for each $i$.

(b) $\Rightarrow$ (a) Let $j>i$. By Applying the exact functor $I \otimes_{\Lambda}-$ to the exact sequence $0 \rightarrow \operatorname{rad}\left({ }_{\Lambda} \Delta(i)\right) \rightarrow{ }_{\Lambda} \Delta(i) \rightarrow{ }_{\Lambda} S(i) \rightarrow 0$, we get the exact sequence $0 \rightarrow I \otimes \operatorname{rad}\left({ }_{\Lambda} \Delta(i)\right) \rightarrow I \otimes{ }_{\Lambda} \Delta(i) \rightarrow I \otimes{ }_{\Lambda} S(i) \rightarrow 0$. Thus, the condition given in (b) implies that $\left[I \otimes{ }_{\Lambda} S(i):{ }_{\Lambda} S(j)\right]=0$; proving (a).

The following result relates the admissibility of $\left({ }_{\Lambda} S, \leq\right)$ with the compatibility of $\left({ }_{\Lambda} \Delta, \leq\right)$.

Proposition 5.8. Let $I_{\Lambda}$ be a projective $\Lambda$-module. Then, the following statements hold true.

(a) If $\left({ }_{\Lambda} S, \leq\right)$ is admissible with the ideal I, then the canonical stratifying system $(\Lambda \Delta, \leq)$ is compatible with the ideal $I$.

(b) If $\Lambda$ is an ss-algebra such that $\left({ }_{\Lambda} \Delta, \leq\right)$ is compatible with the ideal I, then $\left({ }_{\Lambda} S, \leq\right)$ is admissible with the ideal $I$.

Proof. $\quad(a) \Rightarrow$ (b) Assume that, for each $i \in[1, n]$, the $\Lambda$-module $I \otimes_{\Lambda} S(i)$ has composition factors only amongst ${ }_{\Lambda} S(j)$ with $j \leq i$. Then, by [5.7 we get that $\left[I \otimes{ }_{\Lambda} \Delta(j):{ }_{\Lambda} S(i)\right]=0$ for $i>j$. Therefore $\operatorname{Hom}_{\Lambda}\left({ }_{\Lambda} P(i), I \otimes_{\Lambda} \Delta(j)\right)=0$ for $i>j$, and so the condition (C1) in 5.1 holds.

Let now $i \geq j$, and let $\nu: 0 \rightarrow I \otimes{ }_{\Lambda} \Delta(j) \rightarrow U \stackrel{\alpha}{\rightarrow}{ }_{\Lambda} \Delta(i) \rightarrow 0$ be an exact sequence. So, by 5.7 (b), we get that $U \in \mathfrak{F}\left(\left\{{ }_{\Lambda} S(t): t \leq i\right\}\right)$. Consider the epimorphism $p:{ }_{\Lambda} P(i) \rightarrow{ }_{\Lambda} \Delta(i)$ where $\operatorname{Ker}(p)=\operatorname{Tr}_{\oplus_{r>i} \Lambda} P(r)\left({ }_{\Lambda} P(i)\right)$. Then, there is a morphism $f:{ }_{\Lambda} P(i) \rightarrow U$ such that $p=\alpha f$. By taking the factorization ${ }_{\Lambda} P(i) \stackrel{\bar{f}}{\rightarrow} \operatorname{Im}(f) \stackrel{\imath}{\rightarrow} U$ of $f$ throughout its image, we have that $(\alpha \imath) \bar{f}=p$. That is, the quotient morphism $\bar{f}:{ }_{\Lambda} P(i) \rightarrow \operatorname{Im}(f)$ factors throughout $p:{ }_{\Lambda} P(i) \rightarrow{ }_{\Lambda} \Delta(i)$. Moreover, since $\operatorname{Ker}(p)=\operatorname{Tr}_{\oplus r>i} P(r)\left({ }_{\Lambda} P(i)\right)$, it follows that $p$ factors throughout $\bar{f}$. Hence $\alpha \imath: \operatorname{Im}(f) \rightarrow{ }_{\Lambda} \Delta(i)$ is an isomorphism. Therefore, the exact sequence $\nu$ splits, and so the condition (C2) in 5.1 holds.

(b) $\Rightarrow$ (a) Assume that $\Lambda$ is an ss-algebra such that $\left({ }_{\Lambda} \Delta, \leq\right)$ is compatible with the ideal $I$. Let $j>i$ and consider the canonical exact sequence $\eta$ : $0 \rightarrow K(j) \rightarrow{ }_{\Lambda} P(j) \rightarrow{ }_{\Lambda} \Delta(j) \rightarrow 0$, where $K(j):=\operatorname{Tr}_{\oplus_{r>j} \Lambda} P(r)\left({ }_{\Lambda} P(j)\right)$. 
Since $\Lambda$ is an ss-algebra, it is known that $K(j) \in \mathfrak{F}\left(\left\{{ }_{\Lambda} \Delta(t): t>j\right\}\right)$. Thus $\operatorname{Hom}_{\Lambda}\left(K(j), I \otimes{ }_{\Lambda} \Delta(i)\right)=0$ (see $\left.5.1(\mathrm{C} 1)\right)$. Applying $\operatorname{Hom}_{\Lambda}\left(-, I \otimes{ }_{\Lambda} \Delta(i)\right.$ ) to $\eta$, and since $\left({ }_{\Lambda} \Delta, \leq\right)$ is compatible with the ideal $I$, we conclude that $\operatorname{Hom}_{\Lambda}\left({ }_{\Lambda} P(j), I \otimes_{\Lambda} \Delta(i)\right) \simeq \operatorname{Hom}_{\Lambda}\left(K(j), I \otimes_{\Lambda} \Delta(i)\right)=0$. Therefore $\left[I \otimes_{\Lambda} \Delta(i):\right.$ $\left.{ }_{\Lambda} S(j)\right]=0$ for $j>i$. Finally, by 5.7 (b), we conclude that $\left({ }_{\Lambda} S, \leq\right)$ is admissible with the ideal $I$,

Let $I_{\Lambda}$ be a projective $\Lambda$-module. In [25], the authors consider as a main hypothesis that the $\Lambda$-module $I \otimes_{\Lambda} S(i)$ has composition factors only amongst ${ }_{\Lambda} S(j)$ with $j \leq i$. As we have seen in 5.8, under the hypothesis that $\Lambda$ is an ss-algebra, this is equivalent to the compatibility condition (see 5.1) for the canonical stratifying system. Observe that 5.1 is precisely the needed condition to determine when a stratifying system in $\bmod (\Lambda)$ can be extended, throughout the functor $G$, to a stratifying system in $\bmod (\Gamma)$ (see 5.2 ).

Proposition 5.9. Let $I_{\Lambda}$ be a projective $\Lambda$-module and let $(\Theta, \leq)$ be a stratifying system of size $t$ in $\bmod (\Lambda)$, which is compatible with the ideal $I$. Then, $(G \Theta, \leq)$ is a stratifying system of size $t$ in $\bmod (\Gamma)$, and the following statements hold true.

(a) The restriction $\left.F\right|_{\mathfrak{F}(G \Theta)}: \mathfrak{F}(G \Theta) \rightarrow \mathfrak{F}(\Theta)$ is well defined, and it is an exact, faithful and dense functor which reflects indecomposables.

(b) The restriction $\left.G\right|_{\mathfrak{F}(\Theta)}: \mathfrak{F}(\Theta) \rightarrow \mathfrak{F}(G \Theta)$ is well defined, and it is an exact and faithful functor which preserves and reflects indecomposables.

(c) The restriction $\left.F\right|_{\operatorname{Im}\left(\left.G\right|_{\mathfrak{F}(\Theta)}\right)}: \operatorname{Im}\left(\left.G\right|_{\mathfrak{F}(\Theta)}\right) \rightarrow \mathfrak{F}(\Theta)$ is an equivalence of categories, and a quasi-inverse is the restriction $\left.G\right|_{\mathfrak{F}(\Theta)}: \mathfrak{F}(\Theta) \rightarrow$ $\operatorname{Im}\left(\left.G\right|_{\mathfrak{F}(\Theta)}\right)$.

Proof. By 5.2 we know that $(G \Theta, \leq)$ is a stratifying in $\bmod (\Gamma)$.

(a) Let $M \in \mathfrak{F}(G \Theta)$. We prove, by induction on the $G \Theta$-length $\ell_{G \Theta}(M)$, that $F(M) \in \mathfrak{F}(\Theta)$ and $\operatorname{Tor}_{1}^{\Gamma}\left(\Lambda_{\Gamma}, M\right)=0$. If $\ell_{G \Theta}(M)=1$ then $M \simeq G \Theta(i)$ for some $i$. Thus $F(M) \simeq F G \Theta(i) \simeq \Theta(i)$ (see 3.1 (a)) and $\operatorname{Tor}_{1}^{\Gamma}\left(\Lambda_{\Gamma}, M\right) \simeq$ $\operatorname{Tor}_{1}^{\Gamma}\left(\Lambda_{\Gamma}, G \Theta(i)\right)=0$ (see $\left.3.2(\mathrm{a})\right)$.

Let $\ell_{G \Theta}(M)>1$. Then, from [21, Lemma 2.8], there is an exact sequence $\eta: 0 \rightarrow G \Theta(i) \rightarrow M \rightarrow N \rightarrow 0$ in $\mathfrak{F}(G \Theta)$, with $\ell_{G \Theta}(N)=\ell_{G \Theta}(M)-1$. Applying the functor $F$ to $\eta$, we get the exact sequence $\operatorname{Tor}_{1}^{\Gamma}\left(\Lambda_{\Gamma}, G \Theta(i)\right) \rightarrow$ $\operatorname{Tor}_{1}^{\Gamma}\left(\Lambda_{\Gamma}, M\right) \rightarrow \operatorname{Tor}_{1}^{\Gamma}\left(\Lambda_{\Gamma}, N\right) \rightarrow F G \Theta(i) \rightarrow F M \rightarrow F N \rightarrow 0$. By induction we know that $\operatorname{Tor}_{1}^{\Gamma}\left(\Lambda_{\Gamma}, N\right)=0$ and $F N \in \mathfrak{F}(\Theta)$. Thus $F(M) \in \mathfrak{F}(\Theta)$ and $\operatorname{Tor}_{1}^{\Gamma}\left(\Lambda_{\Gamma}, M\right)=0$. In particular, it follows that the restriction $\left.F\right|_{\mathfrak{F}(G \Theta)}$ is well defined and it is also an exact functor. Moreover, by 3.1 it is also a faithful and dense functor which reflects indecomposables.

(b) Since $\Gamma_{\Lambda}$ is projective (see $3.2(\mathrm{a})$ ), it follows that $G: \bmod (\Lambda) \rightarrow$ $\bmod (\Gamma)$ is an exact functor. Hence the restriction $\left.G\right|_{\mathfrak{F}(\Theta)}: \mathfrak{F}(\Theta) \rightarrow \mathfrak{F}(G \Theta)$ is 
well defined. Finally, from 3.1 (b) and (c), we conclude that $G$ is faithful and also preserves and reflects indecomposables.

(c) It follows from (a), (b) and 3.3 (a).

Corollary 5.10. Let $I_{\Lambda}$ be a projective $\Lambda$-module and let $(\Theta, \leq)$ be a stratifying system of size $t$ in $\bmod (\Lambda)$, which is compatible with the ideal $I$. Then, the following conditions are equivalent.

(a) The restriction functor $\left.F\right|_{\mathfrak{F}(G \Theta)}: \mathfrak{F}(G \Theta) \rightarrow \mathfrak{F}(\Theta)$ is an exact equivalence of categories, and its quasi-inverse is the restriction functor $\left.G\right|_{\mathfrak{F}(\Theta)}: \mathfrak{F}(\Theta) \rightarrow \mathfrak{F}(G \Theta)$.

(b) $\operatorname{Im}\left(\left.G\right|_{\mathfrak{F}(\Theta)}\right)=\mathfrak{F}(G \Theta)$.

(c) The class $\operatorname{Im}\left(\left.G\right|_{\mathfrak{F}(\Theta)}\right)$ is closed under extensions in $\bmod (\Gamma)$.

Proof. It follows from 5.9 and the fact that $\mathfrak{F}(G \Theta)$ is the smaller full subcategory of $\bmod (\Gamma)$, which contains $G \Theta$ and is closed under extensions.

Observe that, in general, the class $\operatorname{Im}\left(\left.G\right|_{\mathfrak{F}(\Theta)}\right)$ is not necessarily closed under extensions in $\bmod (\Gamma)$. A sufficient condition for the equality $\operatorname{Im}\left(\left.G\right|_{\mathfrak{F}(\Theta)}\right)=$ $\mathfrak{F}(G \Theta)$ will be given in 5.15 ,

Proposition 5.11. Let $I_{\Lambda}$ be projective, $(\Theta, \leq)$ be a stratifying system of size $t$ in $\bmod (\Lambda)$, which is compatible with the ideal $I$, and let $(\Theta, \underline{Q}, \leq)$ be the Ext-projective stratifying system associated to $(\Theta, \leq)$. Then $\operatorname{Ext}_{\Lambda}^{1}(Q, I \otimes \boldsymbol{\Theta})=0$ if and only if the triple $(G \Theta, G \underline{Q}, \leq)$ is the Ext-projective stratifying system associated to the stratifying system $(G \Theta, \leq)$.

Proof. We assert that $\operatorname{Ext}_{\Gamma}^{1}(G Q(i), G \Theta(j)) \simeq \operatorname{Ext}_{\Lambda}^{1}(Q(i), I \otimes \Theta(j))$ for any $i, j \in[1, t]$. Indeed, since $\operatorname{Ext}_{\Lambda}^{1}(Q(i), \Theta(j))=0$ for any $i, j$, then by 3.2 (b), the assertion follows. Thus, by the above assertion, the implication " $\Leftarrow$ " is clear. Assuming that $\operatorname{Ext}_{\Lambda}^{1}(Q, I \otimes \boldsymbol{\Theta})=0$, we obtain from our assertion that $G Q$ is Ext-projective in $G \Theta$. Furthermore, using the fact that $\left.G\right|_{\mathfrak{F}(\Theta)}$ is an exact functor (see $5.9(\mathrm{~b})$ ), we get that the fundamental sequence $\varepsilon_{i}: 0 \rightarrow$ $K(i) \rightarrow Q(i) \rightarrow \Theta(i) \rightarrow 0$, attached to the system $(\Theta, \underline{Q}, \leq)$ (see $2.2(\mathrm{~b})$ ) gives the fundamental sequence $G \varepsilon_{i}: 0 \rightarrow G K(i) \rightarrow G \bar{Q}(i) \rightarrow G \Theta(i) \rightarrow 0$ corresponding to the system $(G \Theta, G \underline{Q}, \leq)$.

Theorem 5.12. Let $I_{\Lambda}$ be projective, $(\Theta, \leq)$ be a stratifying system of size $t$ in $\bmod (\Lambda)$, which is compatible with the ideal $I$ and $\operatorname{Ext}_{\Lambda}^{1}(Q, I \otimes \boldsymbol{\Theta})=0$, and let $(\Theta, \underline{Q}, \leq)$ be the Ext-projective stratifying system associated to $(\Theta, \leq)$. Consider the algebras $A:=\operatorname{End}_{\Lambda}(Q)^{o p}$ and $G A:=\operatorname{End}_{\Gamma}(G Q)^{o p}$, where $Q:=$ $\oplus_{i=1}^{t} Q(i)$. Then, the following statements hold true.

(a) $G A$ is an split-by-nilpotent extension of $A$ by $\operatorname{Hom}_{\Lambda}(Q, I \otimes Q)$. Furthermore, both algebras $A$ and $G(A)$ are basic, standardly stratified and $r k K_{0}(A)=t=r k K_{0}(G A)$. 
(b) $\bar{G}:=\left.\operatorname{Hom}_{\Gamma}(G Q,-) \circ G\right|_{\mathfrak{F}(\Theta)} \circ Q \otimes_{A}-: \mathfrak{F}\left({ }_{A} \Delta\right) \rightarrow \mathfrak{F}\left(G_{A} \Delta\right)$ is well defined and it is an exact and faithful functor which preserves and reflects indecomposables, and $\bar{G}\left({ }_{A} \Delta(i)\right) \simeq{ }_{G A} \Delta(i)$ for any $i \in[1, t]$.

(c) $\bar{F}:=\left.\operatorname{Hom}_{\Lambda}(Q,-) \circ F\right|_{\mathfrak{F}(G \Theta)} \circ G Q \otimes_{G A}-: \mathfrak{F}\left({ }_{G A} \Delta\right) \rightarrow \mathfrak{F}\left({ }_{A} \Delta\right)$ is well defined and it is an exact, dense and faithful functor which reflects indecomposables, and $\bar{F}\left({ }_{G A} \Delta(i)\right) \simeq{ }_{A} \Delta(i)$ for any $i \in[1, t]$.

(d) $A$ is quasi-hereditary if and only if $G A$ is quasi-hereditary.

(e) The restriction functor $\left.\bar{F}\right|_{\operatorname{proj}(G A)}: \operatorname{proj}(G A) \rightarrow \operatorname{proj}(A)$ is an equivalence and its quasi-inverse is $\left.\bar{G}\right|_{\operatorname{proj}(A)}: \operatorname{proj}(A) \rightarrow \operatorname{proj}(G A)$.

Proof. (a) It follows from 4.3 and [21, Theorem 3.2 (a)].

(b) By [21, Theorem 3.2 ], it follows that the functors $Q \otimes_{A}-: \mathfrak{F}\left({ }_{A} \Delta\right) \rightarrow$ $\mathfrak{F}(\Theta)$ and $\operatorname{Hom}_{\Gamma}(G Q,-): \mathfrak{F}(G \Theta) \rightarrow \mathfrak{F}\left(G_{A} \Delta\right)$ are exact equivalences. Thus, by 5.9 (b), the functor $\bar{G}:=\left.\operatorname{Hom}_{\Gamma}(G Q,-) \circ G\right|_{\mathfrak{F}(\Theta)} \circ Q \otimes_{A}-: \mathfrak{F}\left({ }_{A} \Delta\right) \rightarrow \mathfrak{F}\left({ }_{G A} \Delta\right)$ is well defined and has the desired properties. Moreover, by [21, Theorem 3.1 ], we have that $Q_{A} \otimes{ }_{A} \Delta(i) \simeq \Theta(i)$ and $\operatorname{Hom}_{\Gamma}(G Q, G Q(i)) \simeq{ }_{G A} \Delta(i)$. Therefore $\bar{G}\left({ }_{A} \Delta(i)\right) \simeq{ }_{G A} \Delta(i)$ for any $i \in[1, t]$.

(c) It follows, as in the proof of (b), from [21, Theorem 3.2] and [5.9 (a).

(d) By [21, Theorem 3.2], it is enough to see that: $\operatorname{rad}\left(\operatorname{End}_{\Lambda}(\Theta(i))\right)=0$ if and only if $\operatorname{rad}\left(\operatorname{End}_{\Gamma}(G \Theta(i))\right)=0$ for any $i \in[1, t]$. By 5.9 (a) and (b), we have the ring morphisms $\operatorname{End}_{\Lambda}(\Theta(i)) \stackrel{G}{\rightarrow} \operatorname{End}_{\Gamma}(G \Theta(i)) \stackrel{F}{\rightarrow} \operatorname{End}_{\Lambda}(\Theta(i))$. Thus $G\left(\operatorname{rad}\left(\operatorname{End}_{\Lambda}(\Theta(i))\right)\right) \subseteq \operatorname{rad}\left(\operatorname{End}_{\Gamma}(G \Theta(i))\right)$ and also we have the inclusion $F\left(\operatorname{rad}\left(\operatorname{End}_{\Gamma}(G \Theta(i))\right)\right) \subseteq \operatorname{rad}\left(\operatorname{End}_{\Lambda}(\Theta(i))\right)$. Therefore, the desired equivalence holds by the fact that $F$ and $G$ are faithful functors.

(e) It follows from 3.3 (b) and [21, Theorem 3.2].

Remark 5.13. Let $(\Theta, \underline{Q}, \leq)$ be an Ext-projective stratifying system in $\bmod (\Lambda)$. Observe that, if $I \otimes \boldsymbol{\Theta} \bar{\in} \mathfrak{F}(\Theta)$ then $\operatorname{Ext}_{\Lambda}^{1}(Q, I \otimes \boldsymbol{\Theta})=0$.

As we have seen above (see 5.9 and 5.10$)$ the restriction functor $\left.G\right|_{\mathfrak{F}(\Theta)}$ : $\mathfrak{F}(\Theta) \rightarrow \mathfrak{F}(G \Theta)$ is not, in general, an equivalence. So, in the following results, we give sufficient conditions ensuring that $\left.G\right|_{\mathfrak{F}(\Theta)}$ is an equivalence of categories. In order to do that, we start with the following lemma.

Lemma 5.14. Let $(\Theta, Q, \leq)$ be an Ext-projective stratifying system of size $t$, in $\bmod (\Lambda)$, such that $\operatorname{Hom}_{\Lambda}(\boldsymbol{\Theta}, I \otimes \boldsymbol{\Theta})=0$. Then, the following statements hold true.

(a) $\operatorname{Hom}_{\Lambda}(M, I \otimes N)=0$ for any $M, N \in \mathfrak{F}(\Theta)$.

(b) $\operatorname{Ext}_{\Lambda}^{1}(Q, I \otimes \boldsymbol{\Theta})=0 \quad \Leftrightarrow \quad \operatorname{Ext}_{\Lambda}^{1}(\boldsymbol{\Theta}, I \otimes \boldsymbol{\Theta})=0$.

Proof. (a) It follows from [21, Lemma 2.8] and by induction on the $\Theta$-length $\ell_{\Theta}(M)$ of $N$.

(b) The implication " $\Leftarrow$ " follows easily since $Q \in \mathfrak{F}(\Theta)$. Let $\operatorname{Ext}_{\Lambda}^{1}(Q, I \otimes$ $\boldsymbol{\Theta})=0$. Then, by applying $\operatorname{Hom}_{\Lambda}(-, I \otimes \Theta(j))$ to the canonical exact sequence $0 \rightarrow K(i) \rightarrow Q(i) \rightarrow \Theta(i) \rightarrow 0$ in $\mathfrak{F}(\Theta)$, we get that $\operatorname{Ext}_{\Lambda}^{1}(\Theta(i), I \otimes$ 
$\Theta(j))=0$ since $\operatorname{Hom}_{\Lambda}(K(i), I \otimes \Theta(j))=0=\operatorname{Ext}_{\Lambda}^{1}(Q(i), I \otimes \Theta(j))$; and hence $\operatorname{Ext}_{\Lambda}^{1}(\boldsymbol{\Theta}, I \otimes \boldsymbol{\Theta})=0$.

Theorem 5.15. Let $I_{\Lambda}$ be projective, $(\Theta, \underline{Q}, \leq)$ be an Ext-projective stratifying system of size t, in $\bmod (\Lambda)$, such that $\operatorname{Hom}_{\Lambda}(\boldsymbol{\Theta}, I \otimes \boldsymbol{\Theta})=0=\operatorname{Ext}_{\Lambda}^{1}(Q, I \otimes \boldsymbol{\Theta})$. Then, the following statements hold true.

(a) The stratifying system $(\Theta, \leq)$ is compatible with $I$, and $(G \Theta, G \underline{Q}, \leq)$ is the Ext-projective stratifying system associated to $(G \Theta, \leq)$.

(b) $\left.G\right|_{\mathfrak{F}(\Theta)}: \mathfrak{F}(\Theta) \rightarrow \mathfrak{F}(G \Theta)$ is an exact equivalence of categories and $\left.F\right|_{\mathfrak{F}(G \Theta)}: \mathfrak{F}(G \Theta) \rightarrow \mathfrak{F}(\Theta)$ is its quasi-inverse.

Proof. By 5.14 (a) we have that $\operatorname{Hom}_{\Lambda}(M, I \otimes N)=0$ for any $M, N \in \mathfrak{F}(\Theta)$. Thus, by 4.2, we conclude that $G=\left.G\right|_{\mathfrak{F}(\Theta)}: \mathfrak{F}(\Theta) \rightarrow \mathfrak{F}(G \Theta)$ is a fully faithful functor. Furthermore, from 5.14 (b), it follows that $\operatorname{Ext}_{\Lambda}^{1}(\boldsymbol{\Theta}, I \otimes \boldsymbol{\Theta})=0$. In particular, the stratifying system $(\Theta, \leq)$ is compatible with $I$. Moreover, from 5.11, we conclude that $(G \Theta, G Q, \leq)$ is the Ext-projective stratifying system associated to $(G \Theta, \leq)$; and hence (a) follows.

In order to prove (b), it is enough to see that $\left.G\right|_{\mathfrak{F}(\Theta)}: \mathfrak{F}(\Theta) \rightarrow \mathfrak{F}(G \Theta)$ is dense. Indeed, if the restriction $\left.G\right|_{\mathfrak{F}(\Theta)}$ is a dense functor, we would have that $\operatorname{Im}\left(\left.G\right|_{\mathfrak{F}(\Theta)}\right)=\mathfrak{F}(G \Theta)$; and so from $[5.10$ we conclude (b).

Finally, we prove that the functor $G=\left.G\right|_{\mathfrak{F}(\Theta)}: \mathfrak{F}(\Theta) \rightarrow \mathfrak{F}(G \Theta)$ is dense. Indeed, let $M \in \mathfrak{F}(G \Theta)$. We proceed by induction on the $G \Theta$-length $\ell_{G \Theta}(M)$. If $\ell_{G \Theta}(M)=1$ then $M \simeq G \Theta(i)$ for some $i$.

Let $\ell_{G \Theta}(M)>1$. Then, by [21, Lemma 2.8], there is an exact sequence $0 \rightarrow G \Theta(i) \rightarrow M \rightarrow M / G \Theta(i) \rightarrow 0$ in $\bmod (\Gamma)$, where $\ell_{G \Theta}(M / G \Theta(i))=$ $\ell_{G \Theta}(M)-1$ for some $i$. So, by induction, there exists $Z \in \mathfrak{F}(\Theta)$ such that $G(Z) \simeq M / G \Theta(i)$. Moreover, by [21, Proposition 2.10], there is an exact sequence $\eta_{Z}: 0 \rightarrow Z^{\prime} \stackrel{u}{\rightarrow} Q_{0}(Z) \stackrel{\varepsilon_{Z}}{\rightarrow} Z \rightarrow 0$ in $\mathfrak{F}(\Theta)$, with $Q_{0}(Z) \in \operatorname{add}(Q)$. Thus, we get the following exact and commutative diagram in $\bmod (\Gamma)$

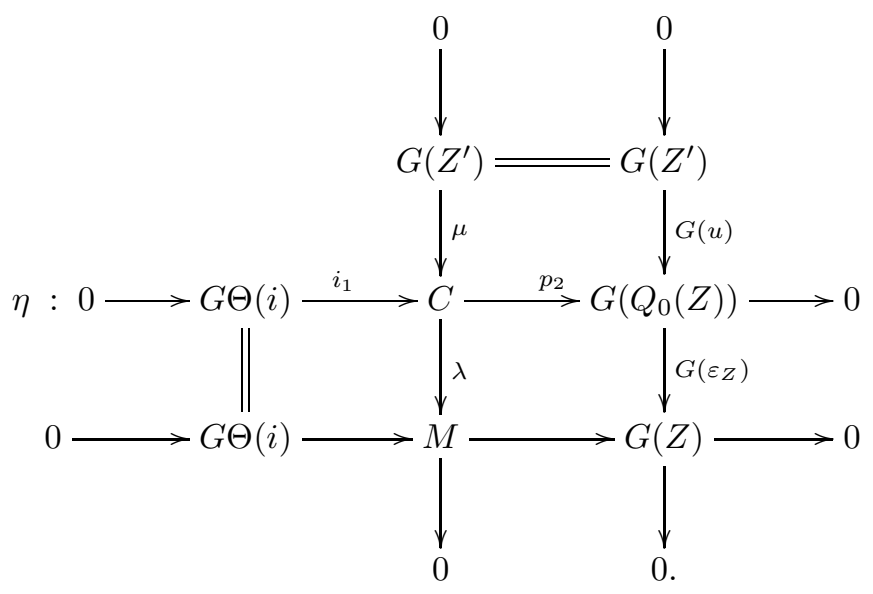


Since $G\left(Q_{0}(Z)\right)$ is Ext-projective in $\mathfrak{F}(G \Theta)$, the exact sequence $\eta$ splits and hence $C=G \Theta(i) \bigoplus G\left(Q_{0}(Z)\right) \simeq G(\Theta(i) \bigoplus G(Z)), i_{1}=\left(\begin{array}{l}1 \\ 0\end{array}\right)$ and $p_{2}=$ $(0,1)$. That is $\mu=\left(\begin{array}{c}\varphi \\ G(u)\end{array}\right)$ with $\varphi: G\left(Z^{\prime}\right) \rightarrow G(\Theta(i))$. Using that the restriction $\left.G\right|_{\mathfrak{F}(\Theta)}$ is full, there exists $h: Z^{\prime} \rightarrow \Theta(i)$ such that $G(h)=\varphi$ and hence $\mu=G(\psi)$, where $\psi:=\left(\begin{array}{c}h \\ u\end{array}\right)$. Observe that the morphism $\psi$ is a monomorphism since $u$ is so. Then, by completing $\psi$ to an exact sequence, we get the following commutative diagram

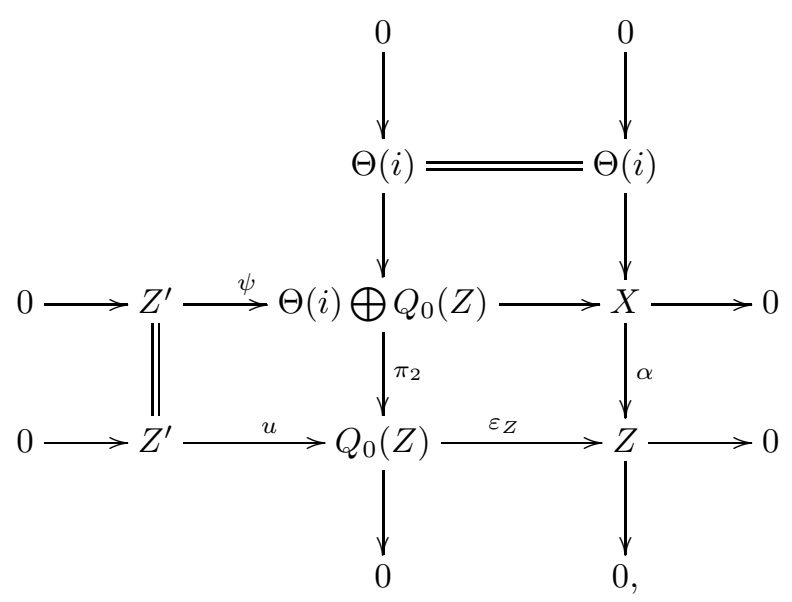

where the rows and columns are exact sequences and $\pi_{2}$ is the canonical projection. Observe that $X \in \mathfrak{F}(\Theta)$ since $\mathfrak{F}(\Theta)$ is closed under extensions. Thus, we get the exact sequence

$$
0 \longrightarrow G\left(Z^{\prime}\right) \stackrel{G(\psi)}{\longrightarrow} G\left(\Theta(i) \oplus Q_{0}(Z)\right) \longrightarrow G(X) \longrightarrow 0
$$

But $G(\psi)=\mu$ and hence $G(X) \simeq \operatorname{Coker}(\mu)=M$; proving that $G: \mathfrak{F}(\Theta) \rightarrow$ $\mathfrak{F}(G \Theta)$ is dense.

Let us consider the following examples. 
Example 5.16. Let $\Gamma$ be the quotient path $k$-algebra given by the quiver

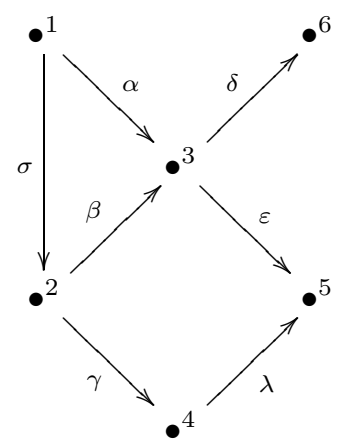

modulo the relations $\delta \beta=0$ and $\varepsilon \beta=\lambda \gamma$. Consider the ideal $I=\langle\bar{\beta}, \bar{\gamma}\rangle \unlhd \Gamma$. So, $\Gamma$ is an split-by-nilpotent extension of $\Lambda:=\Gamma / I$ by $I$. Furthermore, the algebra $\Lambda$ is the path $k$-algebra given by the quiver

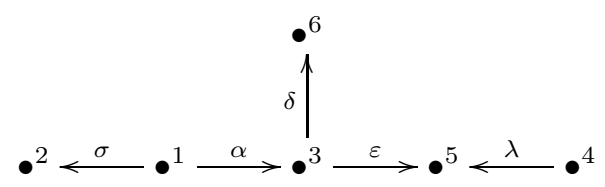

Observe that $I_{\Lambda}$ is projective, since $I_{\Lambda} \simeq{ }_{\Lambda^{o p}} P(2)^{3}$. We consider the natural order $1 \leq 2 \leq 3 \leq 4$ and the stratifying system $(\Theta, \leq)$ of size 4 in $\bmod (\Lambda)$, where $\Theta(1)=\begin{aligned} & S(1) \\ & S(3)\end{aligned}={ }_{\Lambda} I(3), \Theta(2)=\begin{gathered}S(3) S(4) \\ S(5)\end{gathered}, \Theta(3)=S(4), \Theta(4)=S(2)=$ ${ }_{\Lambda} P(2)$. An explicit calculation gives us that $I \otimes \Theta(i)=0$ for $i=1,2,3$ and $I \otimes \Theta(4) \simeq \Theta(2)$. So $I \otimes \Theta \in \mathfrak{F}(\Theta)$ and by 5.13 it follows that $\operatorname{Ext}_{\Lambda}^{1}(Q, I \otimes \Theta)=$ 0 . Moreover, it can be seen that the stratifying system $(\Theta, \leq)$ is compatible with the ideal I. Thus, the needed conditions in 5.12 hold. Finally, observe that $\operatorname{Hom}_{\Lambda}(\Theta, I \otimes \Theta) \neq 0$ since $\operatorname{Hom}_{\Lambda}(\Theta(2), I \otimes \Theta(4)) \simeq \operatorname{End}_{\Lambda}(\Theta(2)) \neq 0$.

Example 5.17. Let $\Gamma$ be the quotient path $k$-algebra given by the quiver

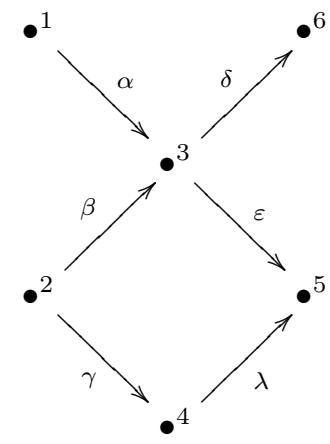


modulo the relations $\delta \beta=0$ and $\varepsilon \beta=\lambda \gamma$. Consider the ideal $I=\langle\bar{\beta}, \bar{\gamma}\rangle \unlhd \Gamma$. So, $\Gamma$ is an split-by-nilpotent extension of $\Lambda:=\Gamma / I$ by $I$. Furthermore, the algebra $\Lambda$ is the path $k$-algebra given by the quiver

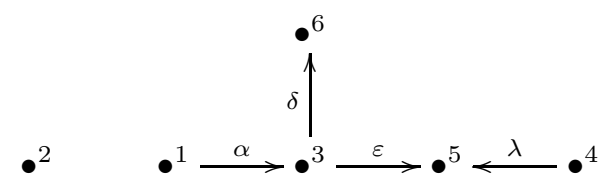

Observe that $I_{\Lambda}$ is projective, since $I_{\Lambda} \simeq \Lambda_{\Lambda^{o p}} P(2)^{3}$. We consider the natural order $1 \leq 2 \leq 3$ and the stratifying system $(\Theta, \leq)$ of size 3 in $\bmod (\Lambda)$, $S(1)$

where $\Theta(1)=S(4) S(3)={ }_{\Lambda} I(5), \Theta(2)=S(2)={ }_{\Lambda} P(2)$ and $\Theta(3)=S(6)=$ $S(5)$

${ }_{\Lambda} P(6)$. An explicit calculation gives us that $I \otimes \Theta(i)=0$ for $i=1,3$ and $N:=I \otimes \Theta(2)=\begin{gathered}S(3) S(4) \\ S(5)\end{gathered}$. It can be seen that $\operatorname{Hom}_{\Lambda}(\boldsymbol{\Theta}, I \otimes \boldsymbol{\Theta})=0=$ $\operatorname{Ext}_{\Lambda}^{1}(\boldsymbol{\Theta}, I \otimes \boldsymbol{\Theta})$. Thus, by 5.14, the needed conditions in 5.15 hold. Finally, observe that $I \otimes \Theta \notin \mathfrak{F}(\Theta)$ since $I \otimes \Theta(2)=N \notin \mathfrak{F}(\Theta)$.

We finish this section by taking into consideration the canonical stratifying system $\left({ }_{\Lambda} \Delta, \leq\right)$. Let the standard $\Lambda$-modules ${ }_{\Lambda} \Delta$ be computed using the pair $\left({ }_{\Lambda} P, \leq\right)$, where ${ }_{\Lambda} P=\left\{{ }_{\Lambda} P(i)\right\}_{i=1}^{n}$ is a representative set of the indecomposable projective $\Lambda$-modules, $n:=r k K_{0}(\Lambda)$ and $\leq$ is a linear order on the set $[1, n]$. By 3.4, we have that ${ }_{\Gamma} P:=G\left({ }_{\Lambda} P\right)$ is a representative set of the indecomposable projective $\Gamma$-modules. So, we compute the standard $\Gamma$-modules ${ }_{\Gamma} \Delta$ by using the pair $\left({ }_{\Gamma} P, \leq\right)$.

Theorem 5.18. Let $I_{\Lambda}$ be projective, and let $\left({ }_{\Lambda} \Delta, \leq\right)$ be compatible with the ideal $I$. Then, the following statements hold true.

(a) $G\left({ }_{\Lambda} \Delta(i)\right) \simeq{ }_{\Gamma} \Delta(i)$ for any $i \in[1, n]$.

(b) $\left.G\right|_{\mathfrak{F}\left({ }_{\Lambda} \Delta\right)}: \mathfrak{F}\left({ }_{\Lambda} \Delta\right) \rightarrow \mathfrak{F}\left({ }_{\Gamma} \Delta\right)$ is an exact and faithful functor which preserves and reflects indecomposables.

(c) $\left.F\right|_{\mathfrak{F}\left({ }_{\Gamma} \Delta\right)}: \mathfrak{F}\left({ }_{\Gamma} \Delta\right) \rightarrow \mathfrak{F}\left({ }_{\Lambda} \Delta\right)$ is an exact, faithful and dense functor which reflects indecomposables.

Proof. By [5.9, we have the stratifying system $\left(G\left({ }_{\Lambda} \Delta\right), \leq\right)$ in $\bmod (\Gamma)$ and also the exact functors $\mathfrak{F}\left({ }_{\Lambda} \Delta\right) \stackrel{G}{\rightarrow} \mathfrak{F}\left(G\left({ }_{\Lambda} \Delta\right)\right) \stackrel{F}{\rightarrow} \mathfrak{F}\left({ }_{\Lambda} \Delta\right)$ satisfying the desired properties as in (b) and (c). It remains to show that $G\left({ }_{\Lambda} \Delta(i)\right) \simeq{ }_{\Gamma} \Delta(i)$ for any $i \in[1, n]$. Indeed, for each $i \in[1, n]$, consider the exact sequence

$$
\eta_{i}: 0 \longrightarrow K(i) \longrightarrow{ }_{\Lambda} P(i) \stackrel{p_{i}}{\longrightarrow}{ }_{\Lambda} \Delta(i) \longrightarrow 0,
$$

where $K(i):=\operatorname{Tr}_{\oplus_{j>i} \Lambda} P(j)\left({ }_{\Lambda} P(i)\right)$. Thus, we get the exact sequence

$$
G\left(\eta_{i}\right): 0 \longrightarrow G(K(i)) \longrightarrow{ }_{\Gamma} P(i) \stackrel{G\left(p_{i}\right)}{\longrightarrow} G\left({ }_{\Lambda} \Delta(i)\right) \longrightarrow 0 .
$$


Let $Z(i):=\operatorname{Tr}_{\oplus_{j>i} P(j)}\left({ }_{\Gamma} P(i)\right)$, and let $\alpha:{ }_{\Gamma} P(j) \rightarrow{ }_{\Gamma} P(i)$, with $j>i$ be any morphism. Then, from 3.4 there exists $\alpha^{\prime}:{ }_{\Lambda} P(j) \rightarrow{ }_{\Lambda} P(i)$ such that $G\left(\alpha^{\prime}\right)=$ $\alpha$; and hence $G\left(p_{i}\right) \alpha=G\left(p_{i} \alpha^{\prime}\right)=0$. Therefore $\operatorname{Im}(\alpha) \subseteq G(K(i))$; proving that $Z(i) \subseteq G(K(i))$. On the other hand, since $K(i):=\operatorname{Tr}_{\oplus_{j>i} \Lambda} P(j)\left({ }_{\Lambda} P(i)\right)$ and $G$ is an exact functor, we get an epimorphism $\oplus_{j>i} P(j)^{m} \rightarrow G(K(i))$, getting us that $G(K(i)) \subseteq Z(i)$.

Corollary 5.19. Let $I_{\Lambda}$ be projective, and let $\left({ }_{\Lambda} \Delta, \leq\right)$ be compatible with the ideal I. Then the following statements hold true.

(a) $\Lambda$ is an standardly stratified (respectively, quasi-hereditary) algebra if and only if $\Gamma$ is so.

(b) Let $\Lambda$ be an standardly stratified algebra such that $\operatorname{Hom}_{\Lambda}\left({ }_{\Lambda} \boldsymbol{\Delta}, I \otimes\right.$ $\left.{ }_{\Lambda} \boldsymbol{\Delta}\right)=0$. Then, the functor $\left.G\right|_{\mathfrak{F}\left({ }_{\Lambda} \Delta\right)}: \mathfrak{F}\left({ }_{\Lambda} \Delta\right) \rightarrow \mathfrak{F}\left({ }_{\Gamma} \Delta\right)$ is an exact equivalence with quasi-inverse $\left.F\right|_{\mathfrak{F}\left(\Gamma_{\Gamma} \Delta\right)}: \mathfrak{F}\left({ }_{\Gamma} \Delta\right) \rightarrow \mathfrak{F}\left({ }_{\Lambda} \Delta\right)$.

Proof. (a) Let $\Lambda$ be a standardly stratified algebra. For each $i \in[1, n]$, consider the exact sequence

$$
\eta_{i}: 0 \longrightarrow K(i) \longrightarrow{ }_{\Lambda} P(i) \stackrel{p_{i}}{\longrightarrow}{ }_{\Lambda} \Delta(i) \longrightarrow 0
$$

where $K(i):=\operatorname{Tr}_{\oplus_{j>i} \Lambda} P(j)\left({ }_{\Lambda} P(i)\right)$. Since $\Lambda$ is an ss-algebra, we have that $\eta_{i}$ lies in $\mathfrak{F}\left({ }_{\Lambda} \Delta\right)$. Thus, by 5.18 (a), we conclude that the exact sequence $G\left(\eta_{i}\right)$ lies in $\mathfrak{F}\left({ }_{\Gamma} \Delta\right)$; proving that $\operatorname{proj}(\Gamma) \subseteq \mathfrak{F}\left({ }_{\Gamma} \Delta\right)$. That is, the algebra $\Gamma$ is standardly stratified.

Assume now that $\Gamma$ is standardly stratified. Then, by [4], it follows that $\mathfrak{F}\left(\Gamma_{\Gamma} \Delta\right)$ is a resolving category. In particular, the exact sequence $G\left(\eta_{i}\right)$ lies in $\mathfrak{F}\left({ }_{\Gamma} \Delta\right)$. Since $\left.F\right|_{\mathfrak{F}\left(\Gamma_{\Gamma} \Delta\right)}: \mathfrak{F}\left({ }_{\Gamma} \Delta\right) \rightarrow \mathfrak{F}\left({ }_{\Lambda} \Delta\right)$ is exact, by applying $F$ to $G\left(\eta_{i}\right)$, we get that the exact sequence $\eta_{i}$ lies in $\mathfrak{F}\left(\Lambda_{\Lambda} \Delta\right.$ ) (see 3.1 (a)); proving that $\operatorname{proj}(\Lambda) \subseteq \mathfrak{F}\left(\Lambda_{\Lambda} \Delta\right)$. That is, the algebra $\Lambda$ is standardly stratified.

The proof that $\Lambda$ is quasi-hereditary if and only if $\Gamma$ is so, can be done as we did in the proof of 5.12 (d). To do so, just replace there $\Theta$ by ${ }_{\Lambda} \Delta$ and use 5.18 (a).

(b) Let $\Lambda$ be a standardly stratified algebra. Then, the triple $\left({ }_{\Lambda} \Delta,{ }_{\Lambda} P, \leq\right)$ is the Ext-projective stratifying system associated to $\left({ }_{\Lambda} \Delta, \leq\right)$. So the result follows from 5.15 .

\section{REstricting STRATIFYing SYStems With The FUnCTOR $F$}

In this section, let $\Gamma$ be a split-by-nilpotent extension of $\Lambda$ by and ideal $I \unlhd \Gamma$. We consider the change of rings functor $F:={ }_{\Lambda} \Lambda_{\Gamma} \otimes_{\Gamma}-: \bmod (\Gamma) \rightarrow$ $\bmod (\Lambda)$. We ask under which conditions a stratifying system in $\bmod (\Gamma)$ can be restricted, through the functor $F$, to a stratifying system in $\bmod (\Lambda)$.

Observe that, by 3.1 (d), we know that $F$ reflects indecomposables. However, the functor $F$, in general, could not preserve indecomposables. A sufficient condition, for the functor $F$, to preserve indecomposability is given in the following proposition. In order to do that, we will need the next lemma. 
Lemma 6.1. Let $\Lambda_{\Gamma}$ be projective. Then, for any $M, N \in \bmod (\Gamma)$, we have the following long exact sequence of $R$-modules

$$
\begin{gathered}
0 \rightarrow \operatorname{Hom}_{\Gamma}\left(M, I \otimes_{\Gamma} N\right) \rightarrow \operatorname{Hom}_{\Gamma}(M, N) \rightarrow \operatorname{Hom}_{\Lambda}(F(M), F(N)) \rightarrow \\
\operatorname{Ext}_{\Gamma}^{1}\left(M, I \otimes_{\Gamma} N\right) \rightarrow \operatorname{Ext}_{\Gamma}^{1}(M, N) \rightarrow \operatorname{Ext}_{\Lambda}^{1}(F(M), F(N)) \rightarrow \operatorname{Ext}_{\Gamma}^{2}\left(M, I \otimes_{\Gamma} N\right) .
\end{gathered}
$$

Proof. Let $M, N \in \bmod (\Gamma)$. We assert that

$$
\operatorname{Ext}_{\Gamma}^{i}\left(M, \Lambda \otimes_{\Gamma} N\right) \simeq \operatorname{Ext}_{\Lambda}^{i}(F(M), F(N)) \text { for any } i \text {. }
$$

Indeed, we get that $\operatorname{Ext}_{\Lambda}^{i}(F(M), F(N)) \simeq \operatorname{Ext}_{\Gamma}^{i}\left(M, \operatorname{Hom}_{\Lambda}\left(\Lambda_{\Lambda} \Lambda_{\Gamma}, F(N)\right)\right)$ since $\Lambda_{\Gamma}$ is projective (see [30, Exercise 9.21]). On the other hand, from 2.7 we have that $\operatorname{Hom}_{\Lambda}\left({ }_{\Lambda} \Lambda_{\Gamma},{ }_{\Lambda} \Lambda_{\Gamma} \otimes_{\Gamma} N\right) \simeq \operatorname{Hom}_{\Lambda}\left({ }_{\Lambda} \Lambda_{\Gamma},{ }_{\Lambda} \Lambda_{\Gamma}\right) \otimes_{\Gamma} N \simeq{ }_{\Gamma} \Lambda_{\Gamma} \otimes_{\Gamma} N$ as $\Gamma$ modules; and so the assertion follows.

Applying the functor $-\otimes_{\Gamma} N$ to the exact sequence of $\Gamma-\Gamma$ bimodules $0 \rightarrow I \rightarrow \Gamma \stackrel{\pi}{\rightarrow} \Lambda \rightarrow 0$, and using the fact that $\operatorname{Tor}_{1}^{\Gamma}\left(\Lambda_{\Gamma},{ }_{\Gamma} N\right)=0$, we get the exact sequence of $\Gamma$-modules $\eta: 0 \rightarrow I \otimes_{\Gamma} N \rightarrow N \rightarrow \Lambda \otimes_{\Gamma} N \rightarrow 0$. Moreover, by applying the functor $\operatorname{Hom}_{\Gamma}(M,-)$ to $\eta$, and the above assertion, we get the desired exact sequence.

Proposition 6.2. Let $\Lambda_{\Gamma}$ be projective and $M \in \bmod (\Gamma)$ be indecomposable. If $\operatorname{Hom}_{\Gamma}\left(M, I \otimes_{\Gamma} M\right)=0=\operatorname{Ext}_{\Gamma}^{1}\left(M, I \otimes_{\Gamma} M\right)$ then $F(M)$ is indecomposable.

Proof. By the assumed conditions and 6.1 we get an isomorphism $\xi$ : $\operatorname{End}_{\Gamma}(M) \rightarrow \operatorname{End}_{\Lambda}(F(M))$ of $R$-modules. An explicit computation of the map $\xi$ gives us that $\xi(f)(\lambda \otimes m)=\lambda \otimes f(m)$ for any $\lambda \in \Lambda, m \in M$ and $f \in \operatorname{End}_{\Gamma}(M)$. Thus, the map $\xi$ is also a ring homomorphism and hence $\operatorname{End}_{\Gamma}(M) \simeq \operatorname{End}_{\Lambda}(F(M))$ as $R$-algebras; proving that $F(M)$ is indecomposable.

Definition 6.3. A stratifying system $(\Psi, \leq)$, of size $t$ in $\bmod (\Gamma)$, is compatible with the ideal $I \unlhd \Gamma$ if the following conditions hold.

(C1) $\operatorname{Ext}_{\Gamma}^{1}\left(\Psi(j), I \otimes_{\Gamma} \Psi(i)\right)=0$ for $j>i$.

(C2) $\operatorname{Ext}_{\Gamma}^{2}\left(\Psi(j), I \otimes_{\Gamma} \Psi(i)\right)=0$ for $j \geq i$.

Theorem 6.4. Let $\Lambda_{\Gamma}$ be projective, and let $(\Psi, \leq)$ be a stratifying system of size $t$ in $\bmod (\Gamma)$, which is compatible with the ideal $I \unlhd \Gamma$. Then, for each $i \in[1, t]$ and any choice of an indecomposable direct summand $\Theta(i)$ of $F(\Psi(i))$, the pair $(\Theta, \leq)$ is a stratifying system of size $t$ in $\bmod (\Lambda)$.

Proof. Let $i \in[1, t]$. Since $\Psi(i) \neq 0$, it follows from 3.1 (b) that $F(\Psi(i)) \neq 0$. Thus $F(\Psi(i))$ has at least one indecomposable direct summand. Let $\Theta(i)$ be a choice of an indecomposable direct summand of $F(\Psi(i))$. Since $(\Psi, \leq)$ is compatible with the ideal $I$, we get from 6.1 that the following conditions hold: (a) $\operatorname{Hom}_{\Lambda}(F(\Psi(j)), F(\Psi(i)))=0$ for $j>i$, and (b) $\operatorname{Ext}_{\Lambda}^{1}(F(\Psi(j)), F(\Psi(i)))=$ 0 for $j \geq i$. Therefore, the same conditions, as in (a) and (b), hold for $\Theta:=$ 
$\{\Theta(i)\}_{i=1}^{t}$ since each $\Theta(i)$ is an indecomposable direct summand of $F(\Psi(i))$. Hence the result follows.

Example 6.5. Let $\Gamma$ be the split-by-nilpotent extension of $\Lambda$ by $I$, which is considered in 5.5, and take the natural order $1 \leq 2 \leq 3$. Consider the pair $(\Psi, \leq)$, where $\Psi(1):={ }_{\Gamma} S(2), \Psi(2):={ }_{\Gamma} P(1)$ and $\Psi(3):={ }_{\Gamma} S(3)$. Observe that $(\Psi, \leq)$ is a stratifying system in $\bmod (\Gamma)$. Furthermore, since $I \otimes_{\Gamma} \Psi(1)=$ $0=I \otimes_{\Gamma} \Psi(2), I \otimes_{\Gamma} \Psi(3) \simeq_{\Gamma} S(1)$ and $\operatorname{id}\left({ }_{\Gamma} S(1)\right) \leq 1$, it can be seen that the pair $(\Psi, \leq)$ is compatible with the ideal $I$; and furthermore $\Lambda_{\Gamma}$ is projective since $\Lambda_{\Gamma} \simeq \Gamma^{\circ o p} P(3) \oplus \Gamma^{o p} P(2)$. Thus by 6.4 it follows that, for any choice of an indecomposable direct summand $\Theta(i)$ of $F(\Psi(i))$, the pair $(\Theta, \leq)$ is a stratifying system of size 3 in $\bmod (\Lambda)$. Since $F(\Psi(1)) \simeq{ }_{\Lambda} P(2), F(\Psi(2)) \simeq$ ${ }_{\Lambda} P(1)$ and $F(\Psi(3)) \simeq{ }_{\Lambda} P(3)$, we see that the restriction of $(\Psi, \leq)$ to $\bmod (\Lambda)$, through the functor $F$, gives only one stratifying system.

\section{REFERENCES}

[1] I. Assem, F. U. Coelho, S. Trepode. The bound quiver of a split extension. J. of algebra and its applications 7 (4), (2008), 405-423.

[2] I. Agoston, V. Dlab, E. Lukács. Stratified algebras. Math. Rep. Acad. Sci. Canada 20 (1) (1998) 22-28.

[3] I. Assem, N. Marmaridis. Tilting modules over split-by-nilpotent extensions. Comm. in algebra 26, (1998), 1547-1555.

[4] I. Agoston, D. Happel, E. Lukacs, L. Unger. Standardly stratified algebras and tilting. J. Algebra 226 (1), (2000), 144-160.

[5] I. Assem, D. Zacharia. On split-by-nilpotent extensions. Colloquium Mathematicum 98 (2), (2003), 259-275.

[6] I. Assem, D. Zacharia. Full embeddings of almost split sequences over split-by-nilpotent extensions. Colloquium Mathematicum 81, (1999), 21-31.

[7] V. Dlab. Quasi-hereditary algebras revisited. An. St. Univ. Ovidius Constanta 4 (1996) 43-54.

[8] E. Cline, B.J. Parshall, L.L. Scott. Stratifying endomorphism algebras. Memoirs of the AMS 591, (1996).

[9] V. Dlab, C.M. Ringel. The Module Theoretical approach to Quasi-hereditary Algebras. Repr. Theory and Related Topics, London Math. Soc. LNS 168 (1992) 200-224.

[10] Donkin, Stephen; Tange, Rudolf. The Brauer algebra and the symplectic Schur algebra. Math. Z. 265 (2010), no. 1, 187-219.

[11] Erdmann, Karin. Stratifying systems, filtration multiplicities and symmetric groups. J. Algebra Appl. 4 (2005), no. 5, 551-555.

[12] K. Erdmann, C. Sáenz. On standardly stratified algebras. Comm. in algebra 31 (7), (2003), 3429-3446.

[13] Erdmann, Karin; Schroll, Sibylle. On Young modules of general linear groups. J. Algebra 310 (2007), no. 1, 434-451.

[14] Hemmer, David J. Symmetric group modules with Specht and dual Specht filtrations. Comm. Algebra 35 (2007), no. 11, 3292-3306.

[15] F. Huard, M. Lanzilotta, O. Mendoza. An approach to the finitistic dimension conjecture. Journal of algebra 319 (9), (2008), 3918-3934.

[16] M. Lanzilotta, E. N. Marcos, O. Mendoza, C. Saenz. On the relative socle for Stratifying Systems. Comm. in Algebra, 38: 5, (2010), 1677-1694.

[17] Lipping Li. Extension algebras of standard modules. Preprint 2012: arXiv:1110.6502v3 
[18] N. Marmaridis. On extensions of abelian categories with applications to ring theory. J. of algebra 156, (1993), 50-64.

[19] Hartmann, Robert; Paget, Rowena. Young modules and filtration multiplicities for Brauer algebras. Math. Z. 254 (2006), no. 2, 333-357

[20] E.N. Marcos, O. Mendoza, C. Sáenz. Stratifying systems via relative simple modules. J. of algebra $280,(2004), 472-487$.

[21] E.N. Marcos, O. Mendoza, C. Sáenz. Stratifying systems via relative projective modules. Comm. in algebra 33, (2005), 1559-1573.

[22] E. N. Marcos, O. Mendoza, C. Sáenz. Applications of Stratifying Systems to the finitistic dimension. Journal of pure and applied algebra 205 (2), (2006), 393-411.

[23] E. N. Marcos, O. Mendoza, C. Sáenz, R. Zuazua. Quadratic forms associated to Stratifying Systems. Journal of algebra 302 (2006) 750-770.

[24] O. Mendoza, C. Sáenz. Tilting Categories with applications to Stratifying Systems. Journal of Algebra 302 (2006) 419-449.

[25] E. N. Marcos, H. Merklen, C. Sáenz. Standardly stratified algebras and lower triangular algebras. Colloquium Mathematicum 93 (2), (2002) 303-311.

[26] O. Mendoza, M. I. Platzeck, M. Verdecchia. C-filtered modules and proper costratifying systems. Journal of Algebra 348 (2011) 276-293

[27] O. Mendoza, C. Sáenz, C. Xi. Homological systems in module categories over preordered sets. Quart. J. Math. 60, (2009), 75-103.

[28] Mohamed, Soud Khalifa. Relative theory in subcategories. Colloq. Math. 117 (2009), no. 1, 29-63.

[29] C.M. Ringel. The category of modules with good filtrations over a quasi-hereditary algebra has almost split sequences. Math. Z. 208 (1991) 209-233.

[30] J. Rotman. An introduction to homological algebra.Academic Press, 1979.

[31] P. Webb. Stratifications and Mackey functors. I: Functors for a single group. Proc. London Math. Soc. 82 (3), (2001), 299-336.

Marcelo Lanzilotta M:

Centro de Matemática (CMAT),

Instituto de Matemática y Estadística Rafael Laguardia (IMERL),

Universidad de la República.

Iguá 4225, C.P. 11400, Montevideo, URUGUAY.

marclan@cmat.edu.uy, marclan@fing.edu.uy

Octavio Mendoza:

Instituto de Matemáticas,

Universidad Nacional Autónoma de México,

Circuito Exterior, Ciudad Universitaria,

México D.F. 04510, MÉXICO.

omendoza@matem. unam.mx

Corina Sáenz:

Departamento de Matemáticas, Facultad de Ciencias,

Universidad Nacional Autónoma de México,

Circuito Exterior, Ciudad Universitaria,

México D.F. 04510, MÉXICO.

ecsv@lya.fciencias.unam.mx 